\title{
Fermented rice bran supplementation ameliorates obesity via gut microbiota and metabolism modification in female mice
}

\author{
Shiro Tochitani, ${ }^{1,2,3,4,5}$ Yoshiteru Maehara, ${ }^{6}$ Takahiro Kawase, ${ }^{7}$ Takamitsu Tsukahara, ${ }^{7}$ Ryoichi Shimizu, ${ }^{8}$

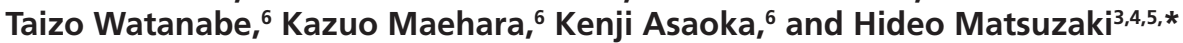

\begin{abstract}
'Division of Health Science, Graduate School of Health Science and ${ }^{2}$ Department of Radiological Technology, Faculty of Health Science, Suzuka University of Medical Science, 3500-3 Minamitamagaki, Suzuka, Mie 513-8670, Japan

${ }^{3}$ Research Center for Child Mental Development, 5 Life Science Innovation Center, and ${ }^{8}$ Faculty of Medical Sciences, University of Fukui, 23-3, Matsuokashimoaizuki, Eiheiji-cho, Yoshida-gun, Fukui 910-1193, Japan

${ }^{4}$ Department of Child Development, United Graduate School of Child Development, Osaka University, 2-2 Yamadaoka, Suita, Osaka 565-0871, Japan ${ }^{6}$ MAX PROBIO. Co. Ltd., 1208, Okaya, Ryuoh-cho, Gamo-gun, Shiga 520-3215, Japan
\end{abstract} \\ ${ }^{7}$ Kyoto Institute of Nutrition \& Pathology, 7-2, Tachikawafuruiketani, Ujidawara-cho, Tsuzuki-gun, Kyoto 610-0231, Japan
}

(Received 29 July, 2021; Accepted 21 August, 2021)

\begin{abstract}
We investigated the effects of fermented rice bran (FRB) administration in two groups of C57BL/6J mice. The first group was fed with a high-fat diet, and the second group was fed with a high-fat diet supplemented with the FRB for 8 weeks. FRB supplementation suppressed the high-fat-induced weight gain and considerable alterations in the intestinal microbiota profile in the second group. Among $\mathbf{2 7}$ bacterial genera detected in the FRB, only Enterococcus, Lactobacillus, Bacteroides, Prevotella, and the unclassified family Peptostreptococcaceae were detected in mice feces. Their abundances were not particularly increased by FRB supplementation. The abundances of Enterococcus and the unclassified family Peptostreptococcaceae were even suppressed in the second group, suggesting that FRB supplementation didn't cause an addition of beneficial microbiome but inhibit the proliferation of specific bacteria. Fecal succinic acid concentration was significantly decreased in the second group and highly correlated with the relative abundances of Turicibacter, Enterococcus, and the unclassified family Peptostreptococcaceae. A significant increase in fumaric acid and a decrease in xylitol, sorbitol, uracil, glutamic acid, and malic acid levels were observed in the peripheral blood of the second group. FRB supplementation counteracted the high-fat-induced obesity in mice by modulating the gut microbiota and the host metabolism.
\end{abstract}

Key Words: high-fat diet, obese, probiotics, succinic acid intervention

$\mathrm{T}$ he history of fermented foods and drinks dates back more than 4000 years. Microorganisms seeded in the environment were used for fermentation and the maturation of fermented foods. ${ }^{(1)}$ Fermentation has initially been a method to preserve foods for a more extended period, although it enhances the nutritional value of foods producing health-promoting components. ${ }^{(2)}$ The functional properties of fermented products are, at least in part, associated with the probiotic content of the products. ${ }^{(2)} \mathrm{A}$ recent study clearly showed that the high-fermented-food diet increased microbiota diversity and decreased inflammatory markers. ${ }^{(3)}$ Rice bran (RB) is a rich source of bioactive components, including dietary fiber and antioxidants, which have the potential to promote gastrointestinal health. ${ }^{(4)} \mathrm{RB}$ is currently available in most regions of the world as a by-product of rice polishing. However, RB consumption by humans is limited. Therefore, RB is discarded or used as animal feed. ${ }^{(5)}$ Fermenta- tion of RB has been used to increase its quality or render it edible for humans as a dietary supplement. Fermented RB (FRB) is such foodstuffs that are more enriched in ingredients such as protein, fiber, and phenolic compounds than the usual raw RB. ${ }^{(6)}$ A previous study reported that FRB exhibits vital activities to improve metabolic syndrome.(6) Metabolic syndrome is defined as a cluster of conditions, such as high blood pressure, obesity, diabetes, and hyperlipidemia, associated with an increased risk of developing cardiovascular disease. ${ }^{(6)}$ Inadequate nutrition habits are essential risk factors for metabolic-syndromerelated disease, and consumption of functional foods such as prebiotics and probiotics may mitigate them. There is a need for new therapeutic approaches to reduce the healthcare cost and better control the conditions associated with metabolic syndrome. Alauddin et al. ${ }^{(6)}$ have shown that chronic supplementation with dual FRB-containing Aspergillus kawachii and lactic acid bacteria significantly reduced food intake, body weight, and epididymal fat mass in stroke-prone spontaneously hypertensive rats. They also suggested that dietary supplementation with FRB can mitigate metabolic syndrome by enhancing serum adiponectin levels and downregulating the transcription factors involved in hepatic glucose and fat metabolism. ${ }^{(6)}$

In contrast, despite reports on the effect of FRB supplementation on the gut microbiome, ${ }^{(7-9)}$ no study has yet investigated the anti-obesity effect of FRB in terms of the gut microbiota. Hence, further studies are required to elucidate the complete physiological effect of FRB and confirm its detailed mechanism of action at the molecular level. Therefore, we conducted this study to evaluate the comparative effects of an FRB food supplement on body weight and gut bacterial metabolism in mice fed with a high-fat diet (HFD). We used the FRB supplement, "MAXPROBIO" produced by MAX PROBIO. Co. Ltd., Japan. This FRB product is a unique probiotic material produced by fermentation using multiple microorganisms, including the six strains Bacillus amyloliquefaciens M4, Bacillus subtilis M5, Bacillus sp. M6, Lactobacillus casei (\#NBRC 15883), Bifidobacterium bifidum (\#NBRC 100015), and Aspergillus oryzae (\#NBRC 6215 ). The enzymes produced by these bacteria degrade $R B$, and the final product of FRB contains abundant valuable components such as peptides, various amino acids, oligosaccharides, and fatty

*To whom correspondence should be addressed.

E-mail: matsuzah@u-fukui.ac.jp 
acids. This FRB supplement would also function as a probiotic containing living multispecies microorganisms. Due to its unique and probable efficacy in improving the intestinal environment, this FRB supplement has been primarily used for pet animals and humans. It has also become a supplement food for racehorses, officially approved by Japan Racing Association. In the present study, we explored the effect of this FRB food supplement on HFD-induced obesity using a mouse model. We analyzed the bacterial contents of the final product of the FRB supplement, followed by analyses on the weight gain, the food consumption rate, the behavioral activity, the alterations in the gut microbiota, and the metabolism of the mice induced by the FRB supplementation.

\section{Materials and Methods}

Ethics statement. The methods followed in this study were conducted under approved ethics guidelines. All animal manipulations were performed in accordance with the National Institute of Health Guide for the Care and Use of Laboratory Animals and were approved by the Animal Research Committee at the University of Fukui.

Preparation of FRB. The FRB food supplements were prepared based on the manufacturing process and provided by MAX PROBIO. Co. Ltd. (Ryuoh, Shiga, Japan). Briefly, FRB was prepared by fermentation using the six bacterial strains Bacillus amyloliquefaciens M4, Bacillus subtilis M5, Bacillus sp. M6, Lactobacillus casei (\#NBRC 15883), Bifidobacterium bifidum (\#NBRC 100015), and Aspergillus oryzae (\#NBRC 6215). In the first stage, $300 \mathrm{~kg}$ of $\mathrm{RB}$ was ground to particles measuring $<1 \mathrm{~mm}$, after which $30 \mathrm{~kg}$ of the six bacterial strain mixture was inoculated at an initial concentration of $10^{10} / \mathrm{g}$ and mixed with RB evenly. In the second stage, $115 \mathrm{~L}$ of $0.06 \%$ (w/w) glucose solution was gradually mixed with $330 \mathrm{~kg}$ of RB containing the six bacterial strain mixture in a $1,000 \mathrm{~L}$ vessel. The RB mixture was then covered with a sheet, and the temperature was confirmed constantly to maintain the product at $<50^{\circ} \mathrm{C}$. Then, the temperature was decreased gradually on a daily basis, so that the product temperature reached $<30^{\circ} \mathrm{C} 1$ month later. In the final stage, after confirming that the temperature reached $<30^{\circ} \mathrm{C}$, the FRB was ground to $1 \mathrm{~mm}$-sized particles and dried for at least 2 weeks until the moisture content reached $<12 \%$. Next, the FRB powder was stored in a low-temperature warehouse at $15^{\circ} \mathrm{C}$ until use. The macronutrients present in FRB were analyzed by Japan Food Research Laboratories (JFRL; Tokyo, Japan) using conventional methods for food analysis. Their proximate compositions are shown in Table 1.

Animals and diets. Female C57BL/6J mice aged 4 weeks were purchased from SLC Japan (Hamamatsu, Japan). Four mice were housed in a single cage in a room maintained at $25^{\circ} \mathrm{C}$ with $50 \%$ relative humidity and a 12/12-h light/dark cycle. The animals were randomly divided into two dietary groups $(n=12$ for each group). The first group (HFD group) was fed with HFD (HFD32; CLEA Japan Inc., Tokyo, Japan), and the second group was fed with HFD supplemented with $0.239 \%$ (w/w) FRB food supplement (HFD + FRB group). The concentration of FRB in HFD was determined as follows. A Japanese woman aged 30-39 years with average body weight (53.7 kg: 2013 National Nutrition and Health Survey conducted by the Ministry of Health, Labour and Welfare, Japan) ingests a spoonful of FRB (weighs as $1.3 \mathrm{~g}: 2.42 \times 10^{-5} \mathrm{~g} / \mathrm{g}$ body weight) according to the manufacturer's instruction. The average daily consumption of the HFD diet of 12-week-old female C57BL6 mice with an average body weight of $21.7 \mathrm{~g}$ is $2.2 \mathrm{~g}$ (data supplied by CLEA Japan Inc.). Considering the bodyweight ratio, $5.25 \times 10^{-4} \mathrm{~g}$ of FRB to $21.7 \mathrm{~g}$ bodyweight of mice is equivalent to the daily ingestion of a woman. We finally decided to apply $0.239 \%(\mathrm{w} / \mathrm{w})$ of FRB to HFD so that a female mouse approximately ingests $5.25 \times 10^{-3} \mathrm{~g}$
Table 1. Macronutorients in FRB

\begin{tabular}{lc}
\hline Nutrients & Amount/100 g \\
\hline Energy & $378 \mathrm{kcal}$ \\
Water & $7.9 \mathrm{~g}$ \\
Protein & $17.6 \mathrm{~g}$ \\
Lipids & $21.4 \mathrm{~g}$ \\
Carbohydrates & $42.2 \mathrm{~g}$ \\
Sugar & $15.3 \mathrm{~g}$ \\
Fibers & $26.9 \mathrm{~g}$ \\
Minerals & $10.9 \mathrm{~g}$ \\
Sodium & $6.1 \mathrm{mg}$ \\
Pottasium & $1,950 \mathrm{mg}$ \\
Calcium & $60.1 \mathrm{mg}$ \\
Iron & $9.44 \mathrm{mg}$ \\
Magnesium & $1,110 \mathrm{mg}$ \\
Copper & $0.82 \mathrm{mg}$ \\
Zinc & $8.98 \mathrm{mg}$ \\
Manganese & $21.8 \mathrm{mg}$ \\
Vitamin A (retinol) & $2 \mu \mathrm{gg}$ \\
$\beta$-Carotene & $22 \mu \mathrm{g}$ \\
Vitamin $\mathrm{B}_{1}$ (thiamine) & $1 \mu \mathrm{gg}$ \\
Vitamin $\mathrm{B}_{2}$ (riboflavin) & $1.39 \mu \mathrm{gg}$ \\
Vitamin $\mathrm{B}_{6}$ & $3.14 \mu \mathrm{gg}$ \\
Vitamin E ( $\alpha$-Tocopherol) & $11.9 \mu \mathrm{gg}$ \\
\hline &
\end{tabular}

of FRB (10 times larger amount of an estimated equivalent) through daily consumption of HFD containing FRB. The macronutrient composition analyses of FRB showed that $100 \mathrm{~g}$ of FRB contains $378 \mathrm{kcal}$ (Table 1), suggesting that $5.25 \times 10^{-3} \mathrm{~g}$ of FRB, which is to be consumed per day by a mouse fed with FRB-containing HFD, has only 19.845 calories. Therefore, we decided to use female mice fed with HFD as the control group. The mice were fed for 8 weeks and allowed free access to food and water. The feed intake and weight gain were measured two times a week. Fecal specimens were collected from mice at three defined time points $(0,14$, and 54 days after the onset of the experiment).

Twenty four-h home cage activity test. After the HFDfeeding period, the mice were tested using a 24-h home cage activity test, which was performed as described previously. ${ }^{(10,11)}$ Briefly, we used a home cage activity monitoring system (O'Hara \& Co., Tokyo, Japan), including a home cage $(29 \times 18 \times 12 \mathrm{~cm})$ and an infrared video camera. A 12/12-h light/dark cycle using LED illumination was programmed (08:00-20:00, light period; 20:00-08:00, dark period). Each mouse was separately housed in a cage by 10:30, and images from each cage were captured at a rate of 1 frame/s for $26 \mathrm{~h}$ from 11:00 to 13:00 of the next day. Moreover, the travel distance per hour was analyzed for 24-h (12:00-12:00 of the next day).

Gut microbiota analysis. Bacterial DNA was extracted from mice feces using a commercial extraction kit (QuickGene-810 system and QuickGene DNA tissue kit; KURABO, Osaka, Japan) as described previously. ${ }^{(12)}$ The microbiota composition of the feces was analyzed by the next-generation sequencing method using the MiSeq platform (Illumina, San Diego, CA) as described previously. ${ }^{(13)}$ Briefly, $25 \mathrm{mg}$ of feces was collected into a sterilized conical tube containing beads; then, $250 \mu \mathrm{l}$ of a tissue lysis buffer contained in the kit was applied to the homogenized digest, and the suspensions were smashed twice at 3,000 rpm for 2 min using Micro Smash MS-100 (Tommy, Tokyo, Japan). Next, $25 \mu \mathrm{l}$ of proteinase K contained in the kit was added to suspensions and incubated at 
$55^{\circ} \mathrm{C}$ for $2 \mathrm{~h}$. After centrifugation $\left(15,000 \times g, 10 \mathrm{~min}, 25^{\circ} \mathrm{C}\right)$, $200 \mu \mathrm{l}$ of the supernatant was transferred to a new microtube to which $180 \mu$ of the lysis buffer contained in the kit was added. After incubation at $70^{\circ} \mathrm{C}$ for $10 \mathrm{~min}, 240 \mu \mathrm{l}$ of ethanol $(99 \% \mathrm{v} / \mathrm{v}$; Sigma-Aldrich Japan, Tokyo, Japan) was added. The bacterial genomic DNA in the suspension was purified using the QuickGene 810 system (KURABO). PCR amplification of 16S rRNA V3-V4 region was conducted using the primers 314F and 805R. Processing of the sequencing data, including quality filtering, chimera check, operational taxonomic unit definition, and taxonomy assignment, was conducted using QIIME1.9.1, USEARCH, and UCHIME as described previously. ${ }^{(14)}$ Alphadiversity metrics (Chaol or Shannon index) were calculated using the QIIME1.9.1 software. Beta-diversity was estimated using the UniFrac metric to calculate the distances between the samples and was visualized by principal coordinate analysis (PCoA).

The total number of bacteria in the feces was measured by real-time PCR analysis. Amplification was performed in a $10-\mu 1$ reaction volume containing $5 \mu \mathrm{l}$ of SYBR premix Ex taq (Takara Bio, Shiga, Japan), $1 \mu$ of DNA extract, and $0.2 \mu \mathrm{mol} / \mathrm{L}$ of each primer. The primer sequences were SRV3-1 primer, 5'-CGGYCCAGACTCCTACGGG-3', and SRV3-2 primer, 5'TTACCGCGGCTGCTGGCAC-3'.(15) The PCR amplification protocol consisted of initial denaturation for $1 \mathrm{~min}$ at $95^{\circ} \mathrm{C}$, followed by 35 cycles of melting, annealing, and extension at $95^{\circ} \mathrm{C}$ for $20 \mathrm{~s}$ and $63^{\circ} \mathrm{C}$ for $30 \mathrm{~s}$, and $72^{\circ} \mathrm{C}$ for $45 \mathrm{~s}$, respectively. The cell numbers of total bacteria were calculated using the Bifidobacterium longum JCM $1217^{\mathrm{T}}$ cell numbers.

Organic acid (OA) measurement. The concentrations of OAs, including short-chain fatty acids (SCFAs), in the feces were measured by ion-exclusion HPLC as described elsewhere. ${ }^{(16)}$ Briefly, samples $(0.1 \mathrm{~g})$ were collected and mixed with $0.2 \mathrm{ml}$ of distilled water. Diluents were mixed with $30 \mu$ of $12 \%$ perchloric acid $(\mathrm{v} / \mathrm{v})$. After centrifugation $\left(13,000 \times \mathrm{g}, 4^{\circ} \mathrm{C}, 10 \mathrm{~min}\right)$, the supernatants were filtered through a $0.45-\mu \mathrm{m}$ cellulose acetate membrane filter (Cosmonice Filter W; Nakalai Tesque, Kyoto, Japan) and degassed by vacuum. The supernatants $(5 \mu \mathrm{l})$ were injected into a SIL-10 autoinjector (Shimadzu, Kyoto, Japan). OAs were separated using two serial OA columns (Shim-pack SCR-102H; Shimadzu) with a guard column (SCR-102HG; Shimadzu) at $45^{\circ} \mathrm{C}$ with isocratic elution $(0.8 \mathrm{ml} / \mathrm{min})$ of $5 \mathrm{mmol} / \mathrm{L} \rho$-toluene sulfonic acid aqueous solution using a solvent delivery pump (LC-10ADvp; Shimadzu) with an online degasser (DGU-12A; Shimadzu). OAs were detected using an electronic conductivity detector (Waters 431; Waters, Milford, MA) after post-column dissociation $(0.8 \mathrm{ml} / \mathrm{min})$ with $5 \mathrm{mmol} / \mathrm{L}$ of $\rho$-toluene sulfonic acid, $20 \mathrm{mmol} / \mathrm{L}$ of bis-Tris, and $100 \mu \mathrm{mol} / \mathrm{L}$ of ethylenediaminetetraacetic acid using a solvent delivery pump (LC-10ADvp; Shimadzu). OAs were quantified using a system controller (CBM-20A; Shimadzu). The related pathways to each OA were examined by surveying the Kyoto Encyclopedia of Genes and Genomes (https:/www.genome.jp/ $\mathrm{kegg} / \mathrm{kegg}$ ja.html).

Serum metabolome and bile acid (BA) analyses. After the home cage activity test, the mice were anesthetized with pentobarbital for serum sampling. Blood samples were collected from the inferior vena cava and centrifuged at $1,000 \times \mathrm{g}$ for $15 \mathrm{~min}$ at $4^{\circ} \mathrm{C}$ to obtain serum. Blood sampling was performed at a period between 14:30 and 15:30. Metabolome analyses of the serum in water-soluble low-molecular-weight metabolites were performed by GC-MS as described elsewhere. ${ }^{(17)}$ Briefly, $50 \mu \mathrm{l}$ of serum samples was suspended in $250 \mu \mathrm{l}$ of methanol-chloroform-water (2.5:1:1) and $5 \mu \mathrm{l}$ of $1 \mathrm{mg} / \mathrm{ml}$ 2-isopropylmalic acid as an internal standard. The samples were subsequently mixed in a shaker at $1,200 \mathrm{rpm}$ at $37^{\circ} \mathrm{C}$ for $30 \mathrm{~min}$ and centrifuged at $16,000 \times \mathrm{g}$ for 5 min at $4^{\circ} \mathrm{C}$. Next, $225 \mu \mathrm{l}$ of the supernatant was mixed with 200 $\mu \mathrm{l}$ of distilled water and vortexed. This was followed by centrifu- gation at $16,000 \times g$ for $5 \mathrm{~min}$ at $4^{\circ} \mathrm{C}$. Then, $250 \mu \mathrm{l}$ of the supernatant was dried under vacuum using a centrifugal evaporator (CVE-2200; Tokyo Rikakikai Co., Ltd., Tokyo, Japan). The dried samples were oximated by mixing with $40 \mu \mathrm{l}$ of $20 \mathrm{mg} / \mathrm{ml}$ methoxyamine hydrochloride (MP Biomedicals Japan Co., Ltd., Tokyo, Japan) dissolved in pyridine (FUJIFILM Wako Chemicals Co., Ltd., Osaka, Japan), and the mixture was shaken at 1,200 $\mathrm{rpm}$ at $30^{\circ} \mathrm{C}$ for $90 \mathrm{~min}$. For derivatization, $20 \mu \mathrm{l}$ of $N$-methyl- $N$ trimethylsilyl trifluoroacetamide (Thermo, Waltham, MA) was added to the oximated samples, and the mixture was shaken at $1,200 \mathrm{rpm}$ at $37^{\circ} \mathrm{C}$ for $45 \mathrm{~min}$. The resultant supernatant was analyzed by GC-MS (GCMS QP2010-Ultra, Shimadzu) within $24 \mathrm{~h}$ of derivatization. The Shimadzu Smart Metabolites Database was used to identify metabolites. For calculating the relative concentration of each metabolite, the area of each peak was divided by the area of the internal control peak. 2Isopropylmalic acid was used as the internal control.

BA concentrations in the serum were measured by LC-MS/MS as described elsewhere with some modifications. ${ }^{(18)}$ Briefly, serum $(100 \mu \mathrm{l})$ was transferred to a $2.0-\mathrm{ml}$ microtube and $15 \mu \mathrm{l}$ of internal standard solution $[5 \mathrm{mmol} / \mathrm{L}$ of 2,3-nordeoxycholic acid (NDCA); Steraloids Inc., Newport, RI] was added and vortexed. Next, ethanol $(1.0 \mathrm{ml})$ was added and vortexed for $10 \mathrm{~min}$. After incubation $\left(30 \mathrm{~min}, 60^{\circ} \mathrm{C}\right)$, the sample was heated $(3 \mathrm{~min}$, $\left.100^{\circ} \mathrm{C}\right)$. Then, it was cooled to room temperature and centrifuged $\left(1,600 \times g, 10 \mathrm{~min}, 4^{\circ} \mathrm{C}\right)$. The resulting supernatant was collected to a new $2.0-\mathrm{ml}$ microtube. Residues were resuspended in $1 \mathrm{ml}$ of ethanol, vortexed, centrifuged, and the supernatant was collected in the same manner as mentioned earlier without centrifugation $\left(11,200 \times g, \quad 1 \mathrm{~min}, 4^{\circ} \mathrm{C}\right)$ twice. The collected supernatant $(\sim 2.9 \mathrm{ml})$ was evaporated using the centrifugal evaporator (CVE-2000; EYELA, Tokyo, Japan), and then the dried extract was resuspended in $0.5 \mathrm{ml}$ of methanol. The extract was purified using an HLB cartridge (Waters) according to the manufacturer's instructions. After purification, the effluents were evaporated using the centrifugal evaporator, and the dried extracts were stored at $-80^{\circ} \mathrm{C}$ until analysis. The dried extracts were resuspended in $0.5 \mathrm{ml}$ of methanol just before analysis. The concentrations of BAs such as cholic acid (CA), alpha-muricholic acid (aMCA), beta-muricholic acid (bMCA), chenodeoxycholic acid (CDCA), deoxycholic acid (DCA), hyodeoxycholic acid (HDCA), ursodeoxycholic acid (UDCA), lithocholic acid (LCA), taurocholic acid (TCA), tauro-alpha-muricholic acid (TaMCA), taurochenodeoxycholic acid (TCDCA), taurodeoxycholic acid (TDCA), taurohyodeoxycholic acid (THDCA), taurolithocholic acid (TLCA), glycocholic acid (GCA), glycochenodeoxycholic acid (GCDCA), glycodeoxycholic acid (GDCA), glycohyodeoxycholic acid (GHDCA), glycoursodeoxycholic acid (GUDCA) and glycolithocholic acid (GLCA) were measured using an ultra-pressure liquid chromatography apparatus equipped with a binary solvent manager, an autosampler, and a column heater and tandem mass spectrometry (Acquity TQD UPLC-MS/MS system; Waters). Chromatographic separation was performed using the Acquity BEH C18 column $2.1 \varphi \times 100 \mathrm{~mm}$ (particle size $1.7 \mu \mathrm{m}$; Waters). The mobile phase, delivered at a flow rate of $0.4 \mathrm{ml} / \mathrm{min}$ at $40^{\circ} \mathrm{C}$, was a gradient of solution $\mathrm{A}$ $(10 \mathrm{mmol} / \mathrm{L}$ of ammonium acetate supplemented with acetonitrile:ultrapure water $=20: 80)$ and solution $\mathrm{B}(10 \mathrm{mmol} / \mathrm{L}$ of ammonium acetate supplemented with acetonitrile:ultrapure water $=80: 20$ ). The gradient was as follows: a flow of $95 \%$ solution $\mathrm{A}$ in $5 \mathrm{~min}$, followed by a gradual decrease to $85 \%$ solution $\mathrm{A}$ in $10 \mathrm{~min}$, then a gradual decrease to $75 \%$ solution A in $5 \mathrm{~min}$ and a gradual decrease to $25 \%$ solution $\mathrm{A}$ in $2 \mathrm{~min}$, followed by maintenance of the latter gradient for $4 \mathrm{~min}$. The total run time of this method was $26 \mathrm{~min}$. Solutes were detected using the tandem quadrupole mass spectrometer (Waters) with a Z-spray ion interface. The system was controlled using the Waters MassLynx mass spectrometry software (Waters). Ionization was achieved 
Table 2. Major bacterial components in fermented rice bran. Six samples from three lots (sample A-F) were analyzed

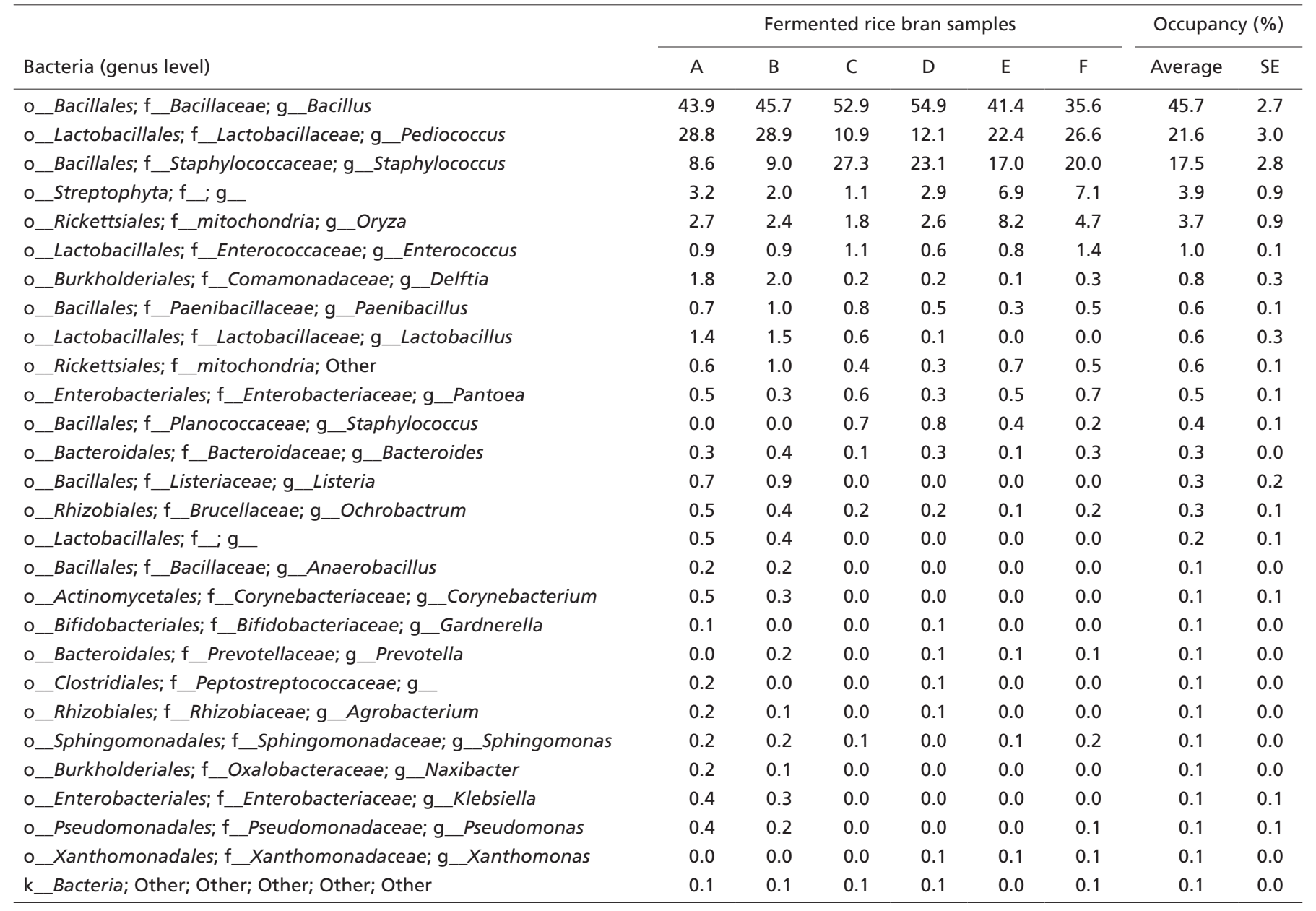

using an alternating electrospray negative ionization mode. The negative ion mode was set as follows: ion source temperature, $150^{\circ} \mathrm{C}$; capillary voltage, $3.0 \mathrm{kV}$; and desolvation temperature, $400^{\circ} \mathrm{C}$. The desolvation and cone gas flow rates were 800 and 50 $\mathrm{L} / \mathrm{h}$, respectively. The selected ion recording (SIR) was performed by examining the product ions of the deprotonated molecules from each BA. Transitions, $\mathrm{m} / \mathrm{z}$ values, and cone voltage were optimized for each BA; each value is shown in Table 2. Dwell time was similar for all BAs $(0.036 \mathrm{~s})$. The injection volume was $5 \mu \mathrm{l}$. The concentrations of individual BAs were calculated from the peak area in the chromatogram detected by SIR relative to the internal standard, NDCA. The related pathways to each metabolite and BA were examined by surveying the Kyoto Encyclopedia of Genes and Genomes (https://www.genome.jp/kegg/kegg_ja.html).

Statistical analysis. Values were expressed as mean $\pm \mathrm{SE}$ or otherwise stated. Statistical analyses were conducted using GraphPad Prism ver. 9.1.2 (GraphPad Software, Inc., La Jolla, CA). A two-tailed unpaired $t$ test, a two-tailed unpaired Welch's corrected $t$ test, or a two-tailed unpaired Mann-Whitney test was used to compare mean values between two groups according to the instructions of GraphPad Prism. In cases where the variances of two groups were significantly different based on the $F$ test result, a two-tailed unpaired Welch's corrected $t$ test was used. If the data set does not pass the Shapiro-Wilk test, a nonparametric test, viz., a two-tailed unpaired Mann-Whitney test was applied. A one-way ANOVA followed by a post hoc Tukey multiple comparisons test was used when comparing three groups. In cases where the variances of groups were significantly different based on the result of the Brown-Forsythe test, Brown-Forsythe and Welch ANOVA tests followed by a post hoc Dunnett's T3 multiple comparisons were used. If the data set does not pass the Shapiro-Wilk test, the Kruskal-Wallis test, followed by a post hoc Dunn's multiple-comparisons test, was used to compare mean values between groups. Values of $p<0.05$ were considered to indicate statistical significance. Pearson's coefficients were calculated to evaluate the correlation between two sets of data.

\section{Results}

FRB contained Bacillus, Pediococcus, Staphylococcus, and other genera as major bacterial components. We first analyzed the primary nutritional composition and the microbial composition of FRB supplements. The final product of FRB contained $378 \mathrm{kcal} / 100 \mathrm{~g}$ energy and a wide variety of nutrients (Table 1). The results of microbial analyses demonstrated that Bacillus, Pediococcus, and Staphylococcus exhibited high abundance in the final product of FRB (Table 2). FRB contained 27 genera as the significant bacterial components (Table 2). The estimated cell numbers in the FRB product were $2.64 \pm 1.09 \times$ $10^{9}$ cells/g FRB. These data were in contrast to the expectation because the FRB was prepared by fermentation initiated by inoculation of the six bacterial strains, Bacillus amyloliquefaciens M4, Bacillus subtilis M5, Bacillus sp. M6, Lactobacillus casei, Bifidobacterium bifidum, and Aspergillus oryzae. 
Effect of chronic supplementation with FRB on diet consumption, body weight, and behavior in HFD-fed mice. We next investigated the effects of FRB on the physiology and behavior of an obese mouse model, which was chronically fed with HFD. We used female mice in this animal model because the prevalence of obesity is higher in women than in men in most countries around the world. ${ }^{(19)}$ Female 6-week-old mice fed with HFD constantly gained weight throughout the observation period (Fig. 1A). The average body weights of mice fed with HFD (HFD) were constantly higher than those of mice fed with HFD supplemented with FRB (HFD + FRB; Fig. 1A). The average alterations in body weight indicated that the body weight gain induced by the HFD diet was moderately suppressed in the HFD + FRB group throughout the observation period (Fig. 1B). The marked suppression of body weight gain was observed during the days after the 38th day of the experiment (Fig. 1B). We next examined the average food consumption of animals, which fluctuated during observation, but we could not identify any apparent difference in food consumption between the two mice groups (Fig. 1C). The calculated averaged food consumption values were $1.25 \mathrm{~g}$ /day in the HFD group and $1.21 \mathrm{~g}$ /day in the HFD + FRB group throughout the experiment. Because the HFD + FRB group was fed with HFD supplemented with $0.239 \%$ (w/w) FRB food supplement, a mouse fed with HFD + FRB daily ingests $2.89 \times 10^{-3} \mathrm{~g}$ of FRB (i.e., $7.63 \times 10^{6}$ bacterial cells/day) on average throughout the experimental period. We next analyzed the spontaneous 24-h locomotor activity of the HFD and HFD + FRB groups on days 56-61 after the start of the experiment (at postnatal 14-week-old) to assess whether the difference in the spontaneous activity between the mice groups affects the body weight gain in the late phase of the observation period. ${ }^{(11)}$ Our results showed no apparent difference in the 24-h spontaneous activity level between the two mice groups (Fig. 2A). A summation of the locomotor activity during the light or dark period also indicated that the spontaneous activity was not different between the HFD and HFD + FRB groups in both periods (Fig. 2B). These results demonstrate that the FRB supplement ameliorates the weight gain induced by HFD, not by affecting the appetite and behavioral activity of the mice.

Effect of chronic supplementation with FRB on the microbial composition in the gut microbiota of HFD-fed mice. As described earlier, a mouse fed with HFD + FRB daily consumes only $2.89 \times 10^{-3} \mathrm{~g}$ of FRB on average. Considering this limited amount of the daily consumption of FRB, the final product of FRB should function mostly as a multispecies probiotic supplement if this type of FRB has any beneficial effects. To elucidate the mechanisms underlying the suppression of weight gain in the mice supplemented with FRB under HFD-feeding conditions, we compared the gut microbiota composition between HFD-fed mice and HFD-fed mice supplemented with FRB at 0,14 , and 54 days after the onset of the experiment. We observed that FRB supplementation did not induce any apparent alteration in the estimated bacterial cell numbers of the gut microbiota detected in feces throughout the experiment (Fig. 3A). Regarding the alphadiversity of the gut microbiota, the values of Chaol indices were significantly decreased at days 14 and 54 after feeding with HFD in both HFD and HFD + FRB groups compared to those at day 0 $(p<0.0001)$, suggesting that HFD feeding affects alpha-diversity. However, we could not find any significant difference between the two groups at any time point (Fig. 3B). The Shannon index values were also significantly decreased by feeding with HFD in both groups $(p<0.0001)$. The Shannon evenness index values only were significantly higher in the HFD + FRB group at day 14 ( $p=0.0166)$ than those in the HFD group. However, there were no significant differences at the other time points (Fig. 3C). These results suggest that, although feeding with HFD decreases the alpha-diversity of the gut microbiota to a great extent, FRB supplementation influences the diversity and evenness of the gut
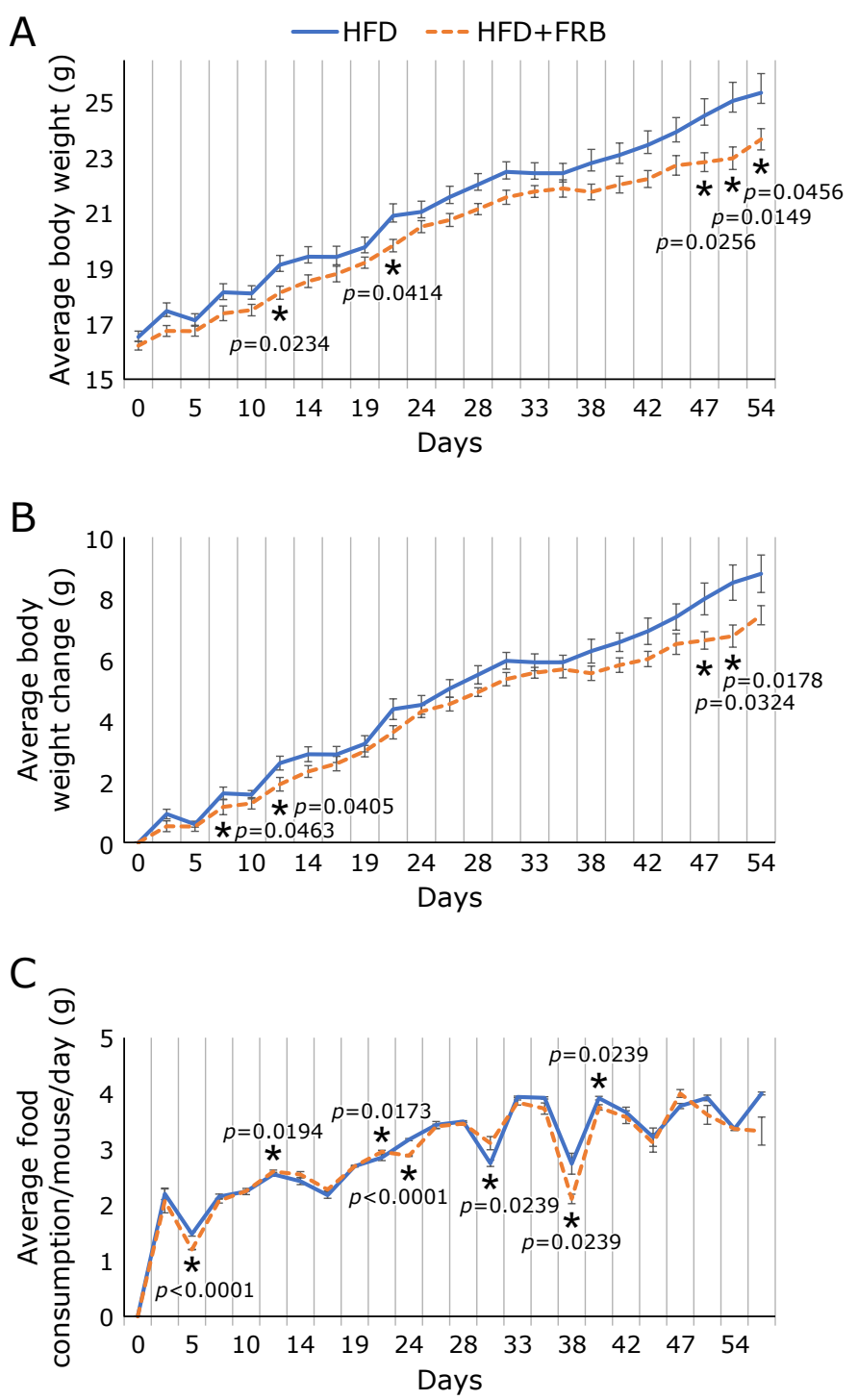

Fig. 1. Bodyweight and food consumption dynamics of mice fed with HFD supplemented with FRB. (A) Average body weight (g) in female mice fed with HFD (HFD) and female mice fed with HFD supplemented with FRB (HFD + FRB) throughout the experiment; HFD, $n=12$ housed in three cages; HFD + FRB, $n=12$ housed in three cages. (B) Average body weight changes per day for a mouse throughout the experiment. (C) Average food consumption (g) per day for a mouse during observation. $P$ values of $<0.05$ are indicated.

microbiota in HFD-fed mice to a limited extent. We next performed PCoA of the beta-diversity of the gut microbiota based on the weighted UniFrac distance on the OTU data (Fig. 3D). Our results demonstrated that the overall composition of the gut microbiota became different from each other throughout the experiment between the HFD and HFD + FRB groups (Fig. 3D). We further evaluated the mean relative abundances of taxa in the HFD and HFD + FRB groups to define the microbiota's specific alterations (Supplemental Table $1 *$ ). At the phylum level, the relative abundance of Bacteroidetes was significantly decreased in both HFD and HFD + FRB groups compared with that at the start of the experiment, whereas the abundance of Bacteroidetes was significantly higher in the HFD + FRB group than in the HFD group at day 14 (Fig. 4A and B). The abundances of Deferribacteres and Proteobacteria tended to increase in both HFD and HFD + FRB groups after the onset of the exper- 


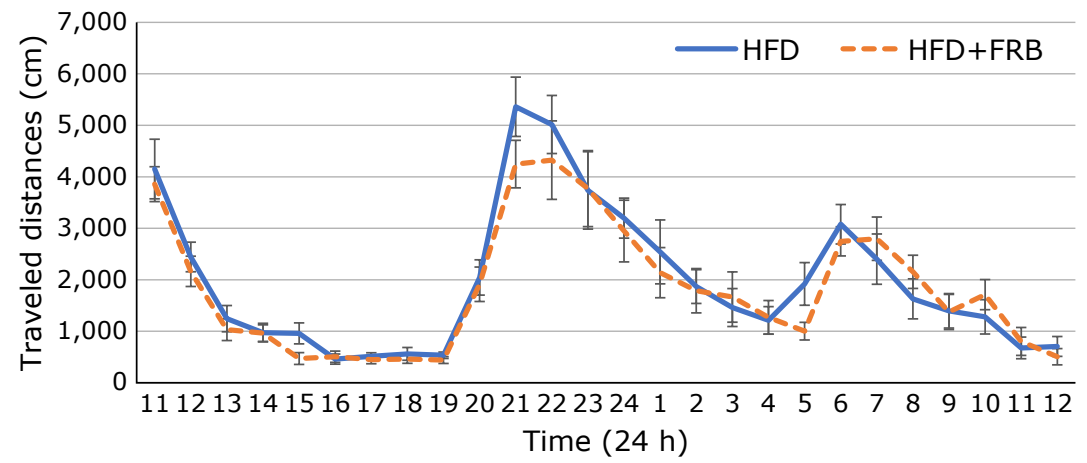

B

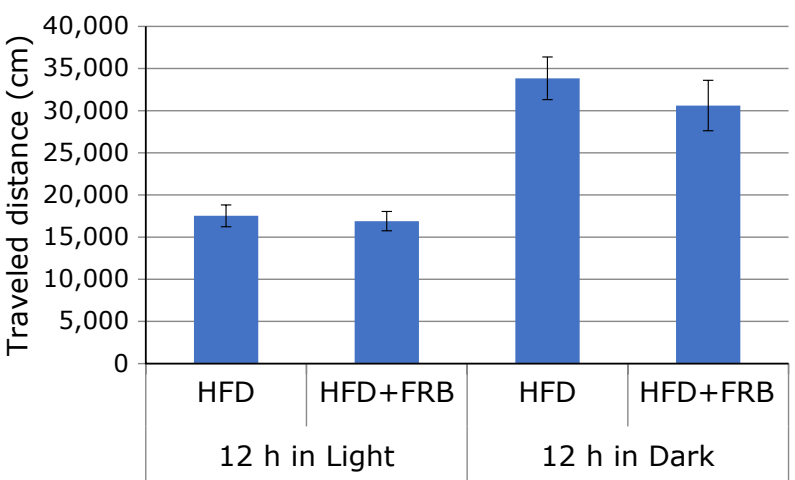

Fig. 2. Behaviors of HFD-fed and HFD + FRB-fed mice at days 56-61 after the experiment (at postnatal 14-week-old). (A) Data for 24-h cycle activity in a familiar environment for HFD-fed and HFD + FRB-fed mice. Throughout the dark phase (20:00 to 8:00) and the light phase (8:00 to 20:00), the mean spontaneous activity of mice housed individually did not show any apparent significant difference between HFD and HFD + FRB feeding. HFD, $n=12$; HFD + FRB, $n=12$. (B) Bar graph showing the averages of summed locomotor activity in the light or dark phase. No significant difference was observed in spontaneous activity between HFD-fed and HFD + FRB-fed mice.

iment (Fig. 4A). At the same time, there were no significant differences in these groups, suggesting that the niche for these phyla was induced by HFD feeding (Fig. 4C and D). The abundance of Firmicutes was constant throughout the experiment (Fig. 4E). It has been reported that the gut microbiota of obese animals and humans exhibits a higher Firmicutes/Bacteroidetes ratio than that of normal-weight individuals. ${ }^{(20-22)}$ Consistent with the observation that HFD-fed mice are more obese than mice fed with HFD + FRB, the Firmicutes/Bacteroidetes ratio transiently became higher in the gut microbiota of the former mice than in the gut microbiota of the latter mice at day 14 (Fig. 4F). At the genus level, we detected a total of 59 classifications and other unclassified bacteria (Supplemental Table $2 *$ ). Regarding the relative abundances of the genera in which the differences between HFD and HFD + FRB groups were observed, we found that FRB supplementation increased the relative abundances of the unclassified order Bacterodales (o_Bacteroidales; $\mathrm{f}_{-} ; \mathrm{g} \_$), the unclassified family Rikenellaceae (o Bacteroidales; $\mathrm{f}$ Rikenellaceae; $\mathrm{g}$ _ ), the unclassified family S24-7 (o_ Bacteroidales; f_S24-7; g_ ), the unclassified family Sphingobacteriaceae - (o_Sphingobacteriales; f Sphingobacteriaceae; Other), and genus Sutterella at day 14 (Fig. 4G-K and N). In particular, the abundance of Sutterella was transiently increased in the HFD + FRB group at day 14 with a statistical significance, but it became similar in both HFD and HFD + FRB groups at day 54 (Fig. 4N). The relative abundance of the unclassified family [Mogibacteriaceae] in the order Clostridiales (o Clostridiales; $f$ [Mogibacteriaceae]; g ) was higher in the HFD + FRB group than in the HFD group at day 54 (Fig. 4L). In contrast, the abundance of the genus [Ruminococcus] in the family Lachnospiraceae (o Clostridiales; f Lachnospiraceae; g_[Ruminococcus]) was significantly lower in the HFD + FRB group at day 54 (Fig. 4M). We detected 27 genera as the major bacterial components in the FRB supplement, as described in Table 2. We next evaluated whether the relative abundances of the genera detected in the FRB supplement were increased in the HFD + FRB group after FRB supplementation. In contrast to the expectation, only five genera (Enterococcus, Lactobacillus, Bacteroides, Prevotella, and the unclassified family Peptostreptococcaceae) among 27 genera in FRB were detected in the gut microbiota of the mice supplemented with FRB (Fig. 5). Remarkably, only Bacteroides exhibited higher abundance in the HFD + FRB group than in the HFD group, particularly at day 14 (Fig. 5C). The relative abundances of Enterococcus and the unclassified family Peptostreptococcaceae were found to be even lower in the HFD + FRB group than those in the HFD group (Fig. 5A and E). The relative abundances of Lactobacillus and Prevotella in the gut microbiota of mice fed with HFD + FRB tended to be similar or slightly lower than those of mice fed with HFD (Fig. 5B and D). We concluded that the observed alterations in the gut microbiota of the HFD + FRB group were induced not only by the addition of microorganisms included in FRB to the gut microbiota of the mice but also by the suppressive effects on the proliferation of specific types of bacteria. The microbial alterations may have occurred due to different microbial interactions and host-microbiota interactions, which the FRB supplementation triggers.

Effect of chronic supplementation with FRB on fecal OA profiles in HFD fed mice. The gut microbiota influences the nutritional acquisition and metabolism of the host. ${ }^{(23)}$ One of 
A

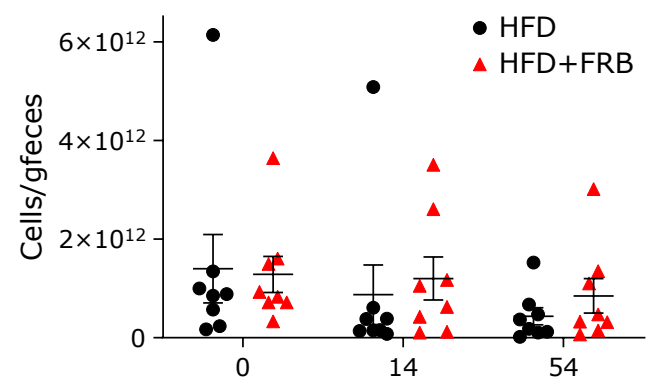

Days after the onset of experiment

C

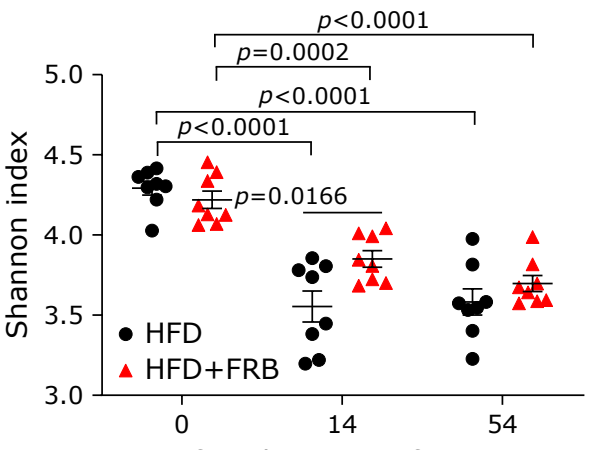

Days after the onset of experiment

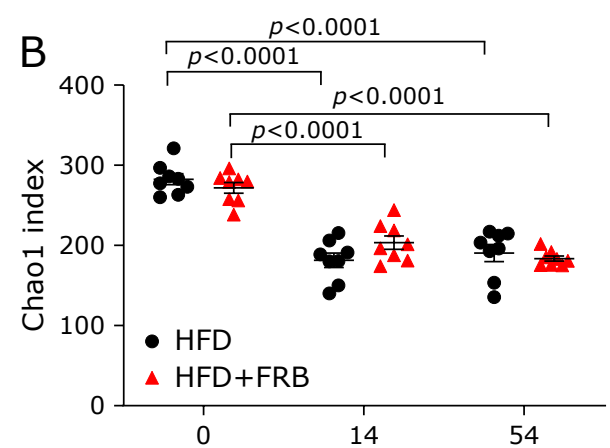

Days after the onset of experiment

D
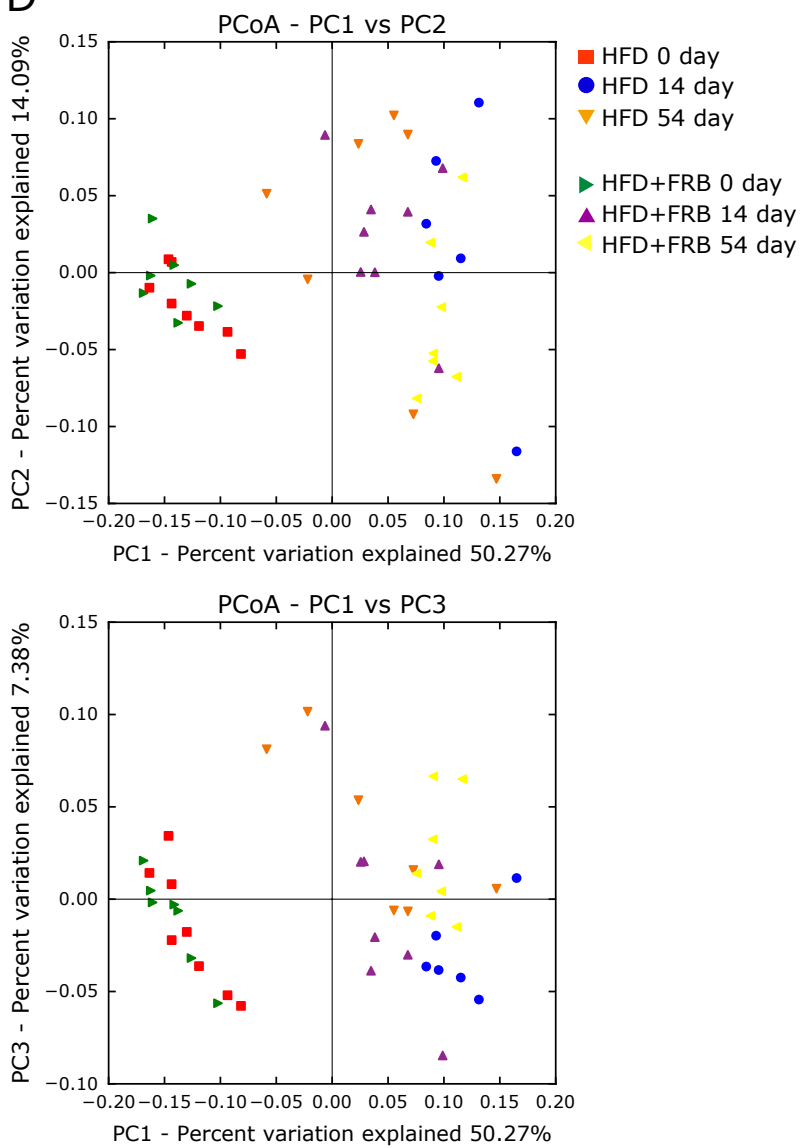

Fig. 3. Effects of administration of the FRB supplement on the gut microbiota of HFD-fed mice. (A) Estimated cell numbers of $16 \mathrm{~S}$ ribosomal RNA genes in fecal samples; $n=8$ for both groups. (B, C) Temporal alteration in alpha-diversity of the gut microbiota based on the Chao1 index (B) and the Shannon index (C) of the OTU level throughout the experiment. $N=8$ for both groups. (D) Principal component analysis of beta-diversity based on the weighted UniFrac analysis of the OTU level. HFD-fed or HFD + FRB-fed mice at each time point are represented in each color as indicated. $P$ values of $<0.05$ are indicated.

the mechanisms by which the gut microbiota affects the host physiology is through SCFAs, which are produced by the gut microbiota. ${ }^{(24-26)}$ SCFAs directly activate the sympathetic nervous system via GPR41, which leads to an increase in energy expenditure. ${ }^{(24,27)}$ GPR43 activation by SCFAs suppresses insulin sensitivity in adipocytes, which inhibits fat accumulation in adipose tissues. ${ }^{(28)}$ Dietary supplementation of SCFAs exhibits preventive effects against diet-induced obesity. ${ }^{(29-31)}$ We then evaluated the concentrations of OAs, including SCFAs, relevant to microbiota in the fecal samples of mice fed with HFD and mice fed with HFD + FRB. We detected the following six OAs (succinic acid, propionic acid, lactic acid, formic acid, acetic acid, and $n$-butyric acid) (Fig. 6A-F). There was a substantial decrease in the fecal succinic acid concentration in the HFD + FRB group at day 54 (Fig. 6A: $p=0.0126$ ). However, there was no significant difference in the concentrations of other OAs between the two groups of mice (Fig. 6B-F: there was $p>0.05$ ). We investigated the bacteria whose abundances correlated well with the concentrations of OAs (Supplemental Table 2*). Results showed that the relative abundance of Turicibacter highly correlated with the fecal concentration of succinic acid $(r=0.9586, p<0.0001$; Fig. 6G). The relative abundance of Turicibacter tended to 
A

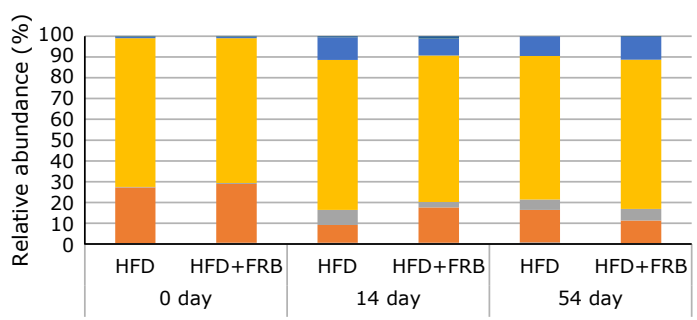

C

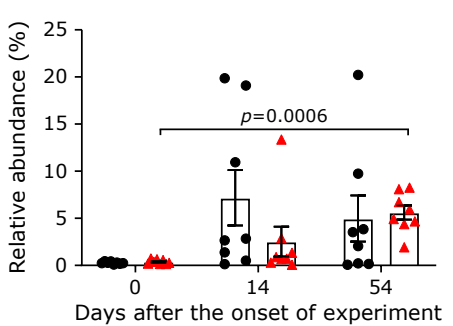

F

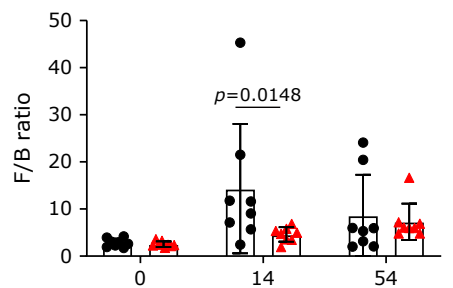

Days after the onset of experiment

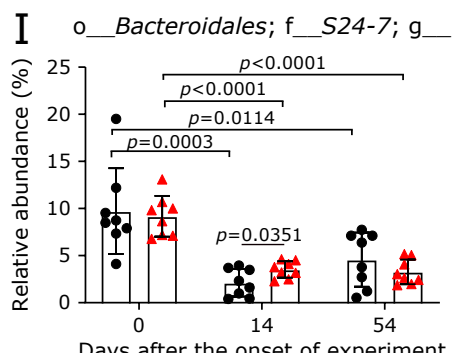

Days after the onset of experiment

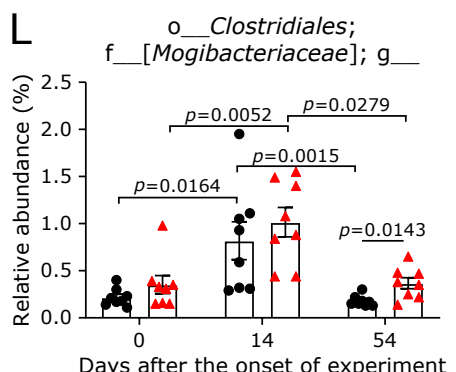

- Actinobacteria - Bacteroidetes - Deferribacteres - Firmicutes - Proteobacteria - Tenericutes - Verrucomicrobia - Other

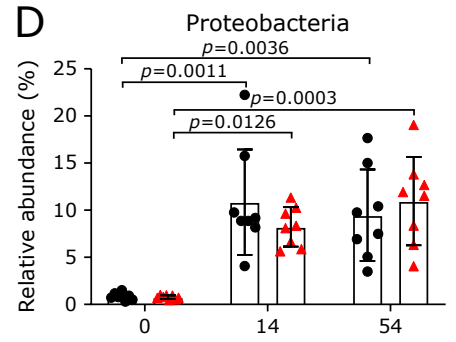

Days after the onset of experiment
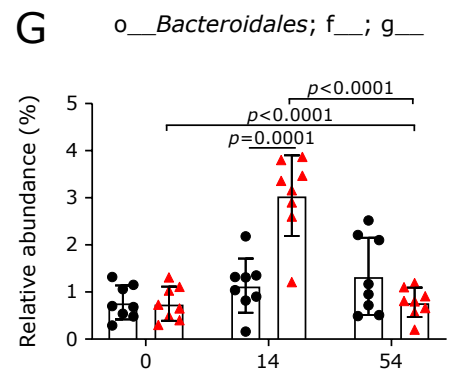

Days after the onset of experiment
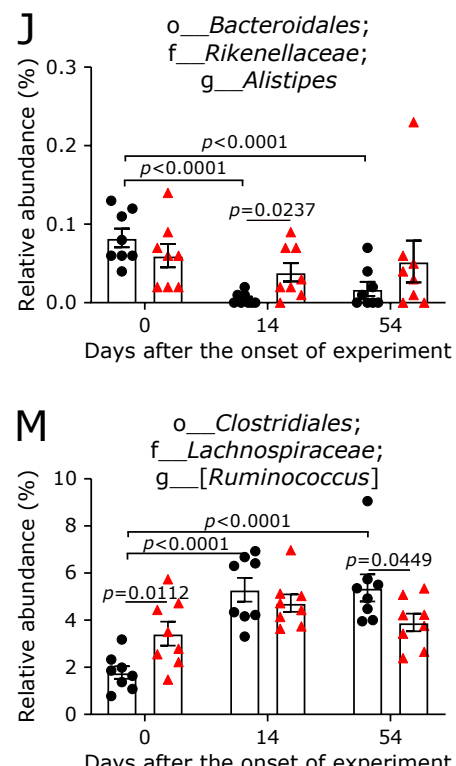
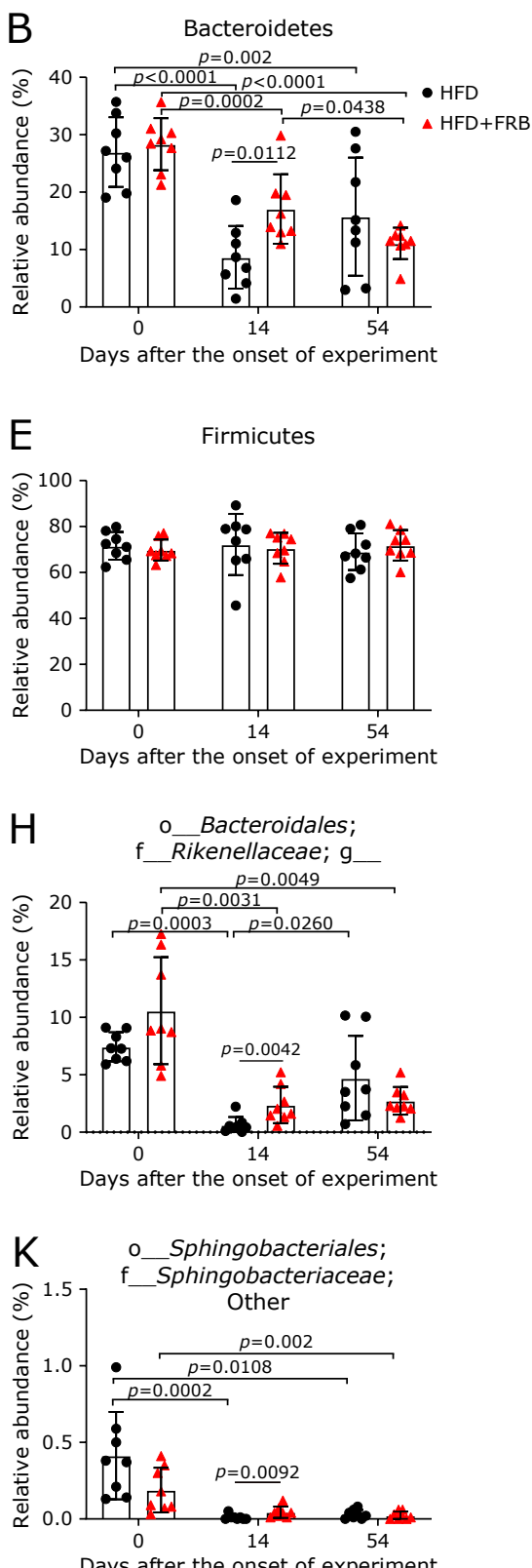

Days after the onset of experiment

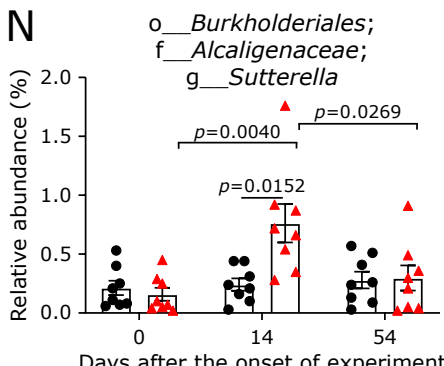

Fig. 4. Alteration in the gut microbiota by FRB supplementation. (A) Abundances of bacteria at phylum level at days 0 , 14 , and 54 after the onset of the experiment. $N=8$ for both groups. (B-E) Relative abundances of Bacteroidetes (B), Deferrubacteres (C), Proteobacteria (D), and Firmicutes (E). (F) The ratio of abundances of Firmicutes/Bacteroidetes. (G-N) Alterations in the gut bacteria at the genus level. Relative abundances of unclassified order Bacteriodales (G), the unclassified family Rikenellaceae (H), unclassified family S24-7 (I), Alistipes (J), the unclassified family Sphingobacteriaceae (K), the unclassified family [Mogibacteriaeae], [Ruminococcus] and Suttella are shown. $P$ values of $<0.05$ are indicated. 

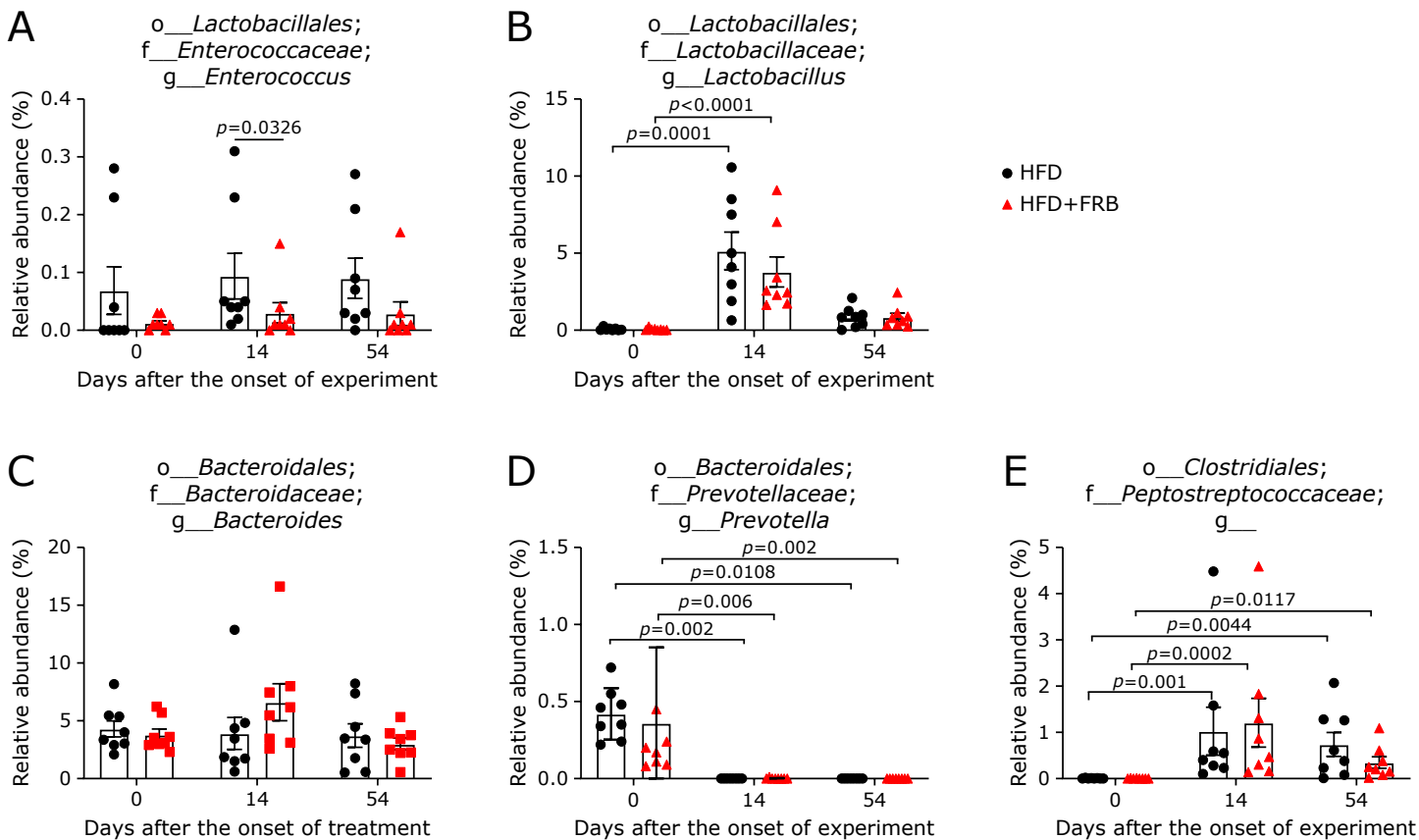

Fig. 5. Temporal alterations in the relative abundances of the bacteria detected in the final product of FRB in the gut microbiota during the experiment period. Relative abundances of Enterococcus (A), Lactobacillus (B), Bacteroides (C), Prevotella (D), and the unclassified family Peptostreptococcaceae are shown. $P$ values of $<0.05$ are indicated. $N=8$ for both groups.

increase after the start of HFD feeding and suppress at days 14 and 54 after FRB supplementation (Fig. 6H). The relative abundances of Enterococcus and the unclassified family Peptostreptococcaceae also correlated with the fecal concentration of succinic acid $(r=0.7795, p=0.0004$, and $r=0.7655, p=0.0005$, respectively; Fig. 6G and Supplemental Table $2 *)$. In particular, these bacteria were detected in the final product of FRB. The relative abundance of Enterococcus was significantly lower in the HFD + FRB mice than in the HFD mice at day 14, and this tendency continued to be observed at day 54 (Fig. 5A). The relative abundance of the unclassified family Peptostreptococcaceae was increased after the onset of HFD-feeding. However, the abundance of Peptostreptococcaceae tended to be lower in the HFD + FRB group than that seen in the HFD group (Fig. 5E). These results suggest that FRB supplementation induced an alteration in the gut microbiota, which led to the suppressed level of succinic acid in the feces.

Effect of chronic supplementation with FRB on serum metabolome and BA profiles in HFD-fed mice. To characterize the peripheral metabolic alteration due to chronic supplementation with FRB during HFD feeding, we designed an experiment to compare serum metabolome and BA profiles between the HFD and HFD + FRB groups. We first conducted a comprehensive metabolome analysis using the serum samples of mice by LC-MS and then detected 82 metabolites (including 2Isopropylmalic acid, which was used as the internal control; Supplemental Table 3*). We investigated the metabolites whose relative concentrations significantly correlated with the weight gain of the mice during days 45-54 after the onset of the experiment. Our results demonstrated that three metabolites (oleic acid, heptadecanic acid, and sorbitol) exhibited positive correlations with weight gain, whereas a metabolite, lyxose, exhibited a negative correlation (Fig. 7A). The relative concentrations of oleic acid, heptadecanic acid, and sorbitol were lower in the serum of mice fed with HFD + FRB than in the serum of mice fed with HFD, although a statistical significance was observed only for sorbitol (Fig. 7B). The concentration of lyxose tended to be higher in the HFD + FRB group than in the HFD group (Fig. 7B). Among the 82 detected metabolites, we identified 9 metabolites that were significantly altered by FRB supplementation (Fig. 7C). We observed that serum fumaric acid concentration was significantly higher in the HFD + FRB group than in the HFD group (Fig. 4A: $p=0.013$ ). The concentrations of malic acid, sorbitol, uracil, xylitol, and glutamic acid were significantly lower in the HFD + FRB group than in the HFD group (Fig. 4B: $p<0.05)$. These nine metabolites whose serum concentrations were significantly altered by FRB supplementation showed no significant correlation with weight gain between days 45 and 54 after the onset of the experiment (Supplemental Table $4 *$ ). Correlation analyses also revealed that the relative abundance of Turicbacter in the fecal bacteria correlated with the relative concentrations of glutamic acid, malic acid, and xylitol (Supplemental Table $4 *$ ). The relative abundances of Enterococcus and the unclassified family Peotostreptococcaceae, both of which are present in the final product of FRB, also correlated with the concentrations of glutamic acid and malic acid (Supplemental Table $4 *$ ). The relative abundance of Butyricoccus positively correlated with the serum concentration of lyxose but negatively with the serum concentration of uracil (Supplemental Table 4*).

We next conducted a BA analysis targeting $20 \mathrm{BAs}$ in the serum samples of mice by LC-MS/MS and then detected 9 BAs (Fig. 8 and Supplemental Table $5 *$ ). None of the detected BAs showed any correlation with bodyweight increase between days 45 and 54 (Supplemental Table 5*). Regarding the primary BAs in mice, ${ }^{(32)}$ there was no difference in the serum concentration of CA, CDCA, aMCA, and bMCA between the HFD and HFD + FRB groups (Fig. 8A). The concentration of UDCA was lower in the HFD + FRB group than in the HFD group, although this effect did not reach statistical significance (Fig. 8A; $p=0.0608$ ). Regarding the secondary BAs in mice, no difference was observed in the serum concentrations of DCA, LCA, and GDCA between the HFD and HFD + FRB groups (Fig. 8B). The concentration of HDCA was lower in the HFD + FRB group than in the HFD group, although the difference was not statistically signifi- 

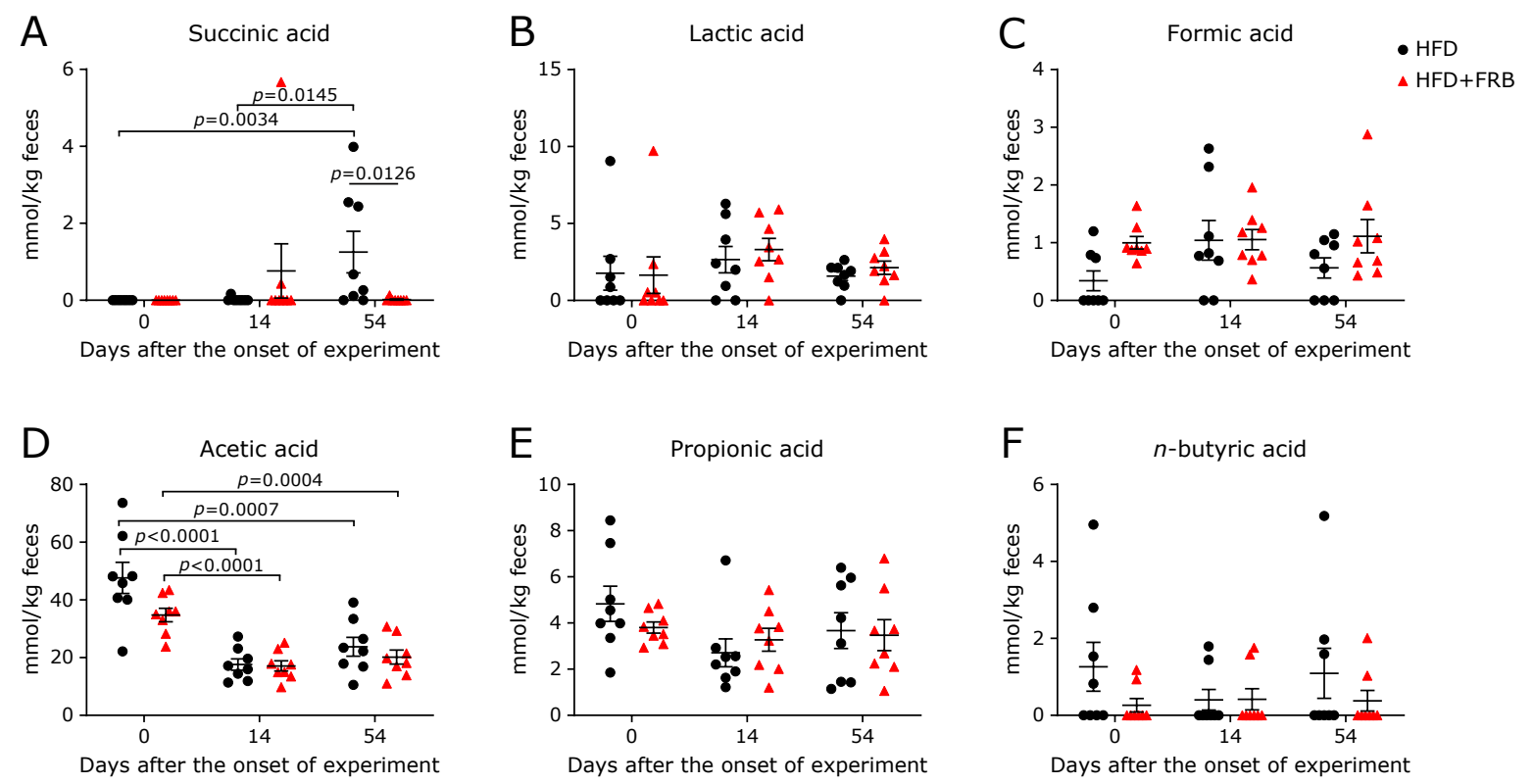

\begin{tabular}{|c|c|c|c|}
\hline \multirow[b]{2}{*}{ Bacteria (genus level) } & \multicolumn{3}{|c|}{ Pearson correlation } \\
\hline & $r$ & Confidence interval & $p$ value \\
\hline o__Turicibacterales; $f$ _Turicibacteraceae; $g$ _ Turicibacter & 0.9586 & 0.8820 to 0.9858 & $<0.0001$ \\
\hline o__Lactobacillales; $f$ _Enterococcaceae; g_Enterococcus & 0.7795 & 0.4624 to 0.9198 & 0.0004 \\
\hline o__Clostridiales; f_Peptostreptococcaceae; $g$ & 0.7655 & 0.4349 to 0.9143 & 0.0005 \\
\hline o__Clostridiales; $f$ _Clostridiaceae; g_Clostridium & 0.6467 & 0.2222 to 0.8651 & 0.0068 \\
\hline C_Bacilli; Other; Other; Other & 0.6340 & 0.2017 to 0.8596 & 0.0084 \\
\hline o__Bacteroidales; $f$ _Rikenellaceae; $g$ & 0.5065 & 0.01448 to 0.8011 & 0.0453 \\
\hline o_Enterobacteriales; $f$ _Enterobacteriaceae; g_Escherichia & 0.5060 & 0.01380 to 0.8009 & 0.0455 \\
\hline o__Clostridiales; $f$ __Ruminococcaceae; g_Oscillospira & -0.6207 & -0.8537 to -0.1805 & 0.0103 \\
\hline o__Clostridiales; $f$ _Christensenellaceae; $g$ __ & -0.5821 & -0.8365 to -0.1215 & 0.018 \\
\hline o__Clostridiales; f_Lachnospiraceae; g_Dorea & -0.5611 & -0.8268 to -0.09060 & 0.0237 \\
\hline
\end{tabular}

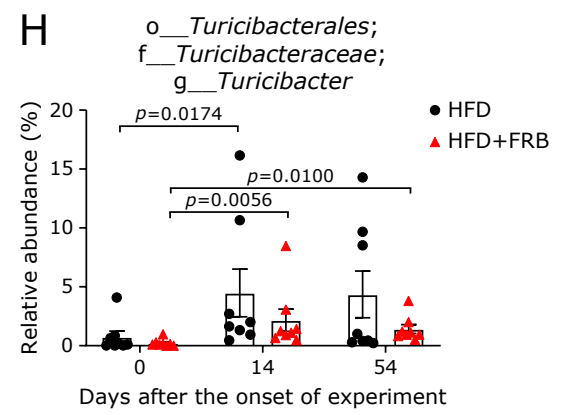

Fig. 6. Alterations in the fecal concentrations of organic acids, including short-chain fatty acids. (A-F) Temporal alterations in the fecal concentration of succinic acid (A), lactic acid (B), formic acid (C), acetic acid (D), propionic acid (E), and $n$-butyric acid (F) in HFD-fed mice and HFD + FRB-fed mice at day 0,14 , and 54 after the onset of the experiment. $P$ values of $<0.05$ are indicated. $N=8$ for both groups. (G) List of bacteria that highly correlated with the fecal concentration of succinic acid. $P$ values of $<0.05$ are indicated. $(\mathrm{H})$ Temporal alterations in the relative abundances of Turicibacter, which increased by HFD feeding and was suppressed by FRB supplementation. $P$ values of $<0.05$ are indicated. $N=8$ for both groups.

cant (Fig. 8B; $p=0.0644)$. Correlation analyses revealed the presence of an association between the relative abundance of Enterococcus in the gut microbiota and the concentration of UDCA, aMCA, and HDCA (Supplemental Table 5*). Overall, these results suggest that the alteration in the gut microbiota induced by FRB supplementation resulted in the peripheral metabolic profiles in the mice.

\section{Discussion}

We explored the effect of FRB supplementation on the body weight and metabolism of female mice under an HFD-fed condition. Supplementation with FRB induced a considerable alteration in the gut microbiota, followed by an increase in the fecal concentration of succinic acid, whose concentration exhibited a high correlation with the relative abundances of Turicibacter, Enterococcus, and the unclassified family Peptostreptococcaceae. Serum metabolome analyses demonstrated that the peripheral 
A

\begin{tabular}{|l|c|c|c|}
\hline & \multicolumn{3}{|c|}{ Pearson correlation (vs weight gain) } \\
\hline \multicolumn{1}{|c|}{ Metabolite } & $r$ & Confidence interval & $p$ value \\
\hline \hline Oleic acid & 0.7238 & 0.3881 to 0.8899 & 0.0007 \\
Heptadecanic acid & 0.5655 & 0.1340 to 0.8167 & 0.0144 \\
\hline Sorbitol & 0.4774 & 0.01355 to 0.7722 & 0.0451 \\
\hline Lyxose & -0.5201 & -0.7941 to -0.07032 & 0.0269 \\
\hline
\end{tabular}

B

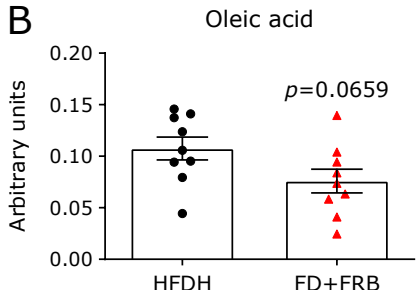

C
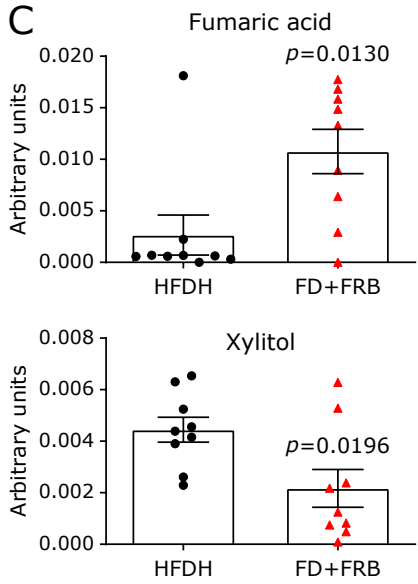

Heptadecanic acid

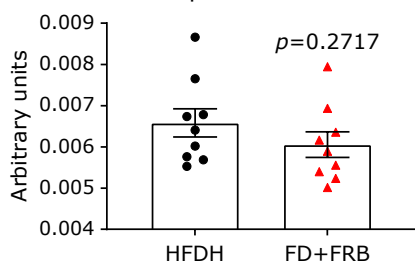

Glutamic acid (3TMS)
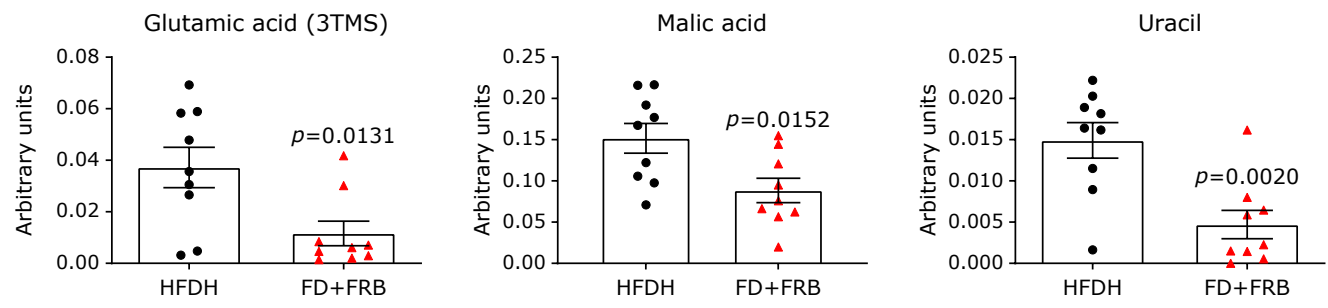

Fig. 7. Comparison of serum metabolome profiles at days 57-61 after the start of the experiment. (A) The relative concentrations of metabolites showing high correlation with body weight gain at days $45-54$. Correlations with $p$ values of $<0.05$ are indicated. The relative concentrations of 82 metabolites detected in the analyses are shown in Supplemental Table 3*. (B) Comparisons of the relative concentrations of the metabolites listed in (A) between HFD-fed and HFD + FRB-fed mice. (C) Comparisons of the relative concentrations of the metabolites between HFD-fed and HFD + FRB-fed mice with statistically significant differences. $N=9$ for both groups.

A Colic acid (CA)
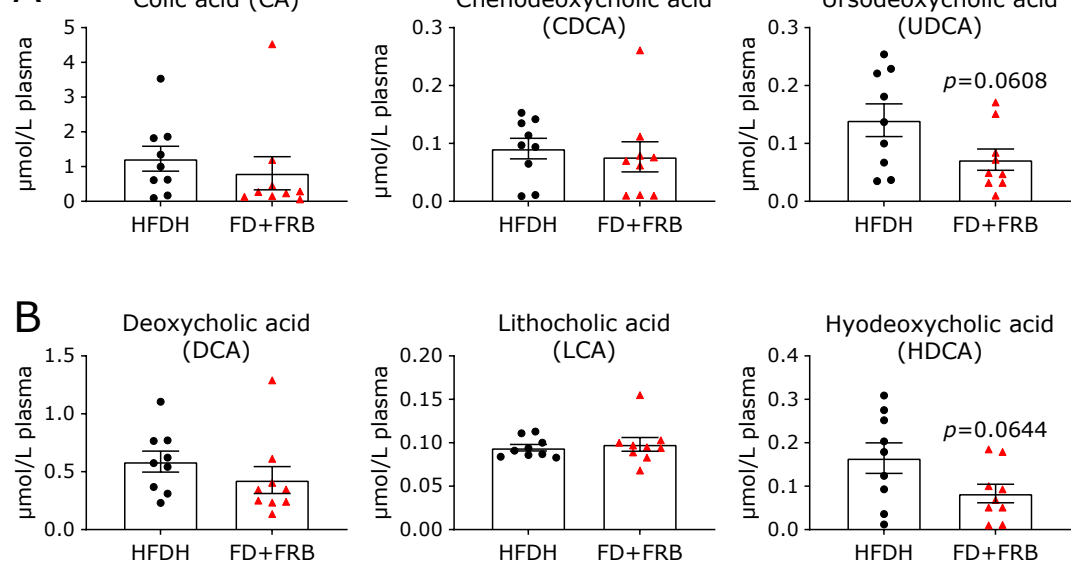
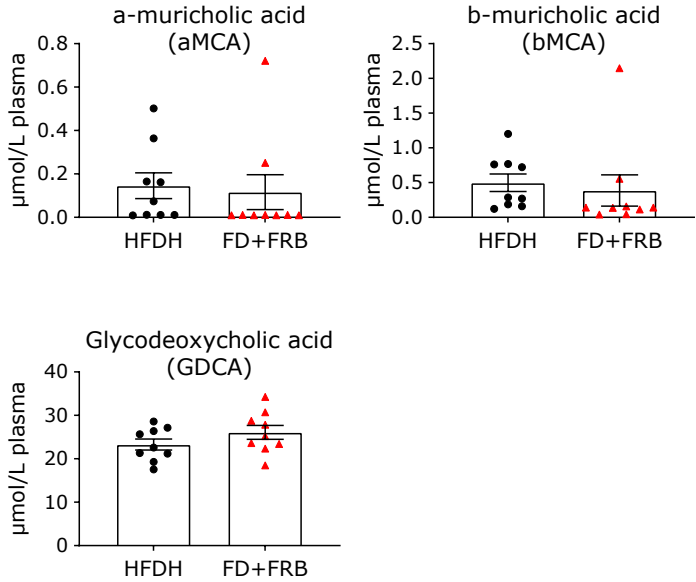

Fig. 8. Comparison of bile acid (BA) profiles in the peripheral blood at days 57-61 after the onset of the experiment. (A) Comparisons of the relative concentrations of primary bile acids between HFD-fed and HFD + FRB-fed mice. (C) Comparisons of the relative concentrations of secondary bile acids between HFD-fed and HFD + FRB-fed mice. $P$ values mentioned in the text are indicated. $N=9$ for both groups. 
metabolome profile had been considerably changed. The serum concentration of fumaric acid was increased in the HFD + FRB group compared with that in the HFD group. The concentrations of sorbitol, glutamic acid, malic acid, uracil, and xylitol were decreased in the HFD + FRB group than in the HFD group. The serum concentrations of some of these metabolites are associated with the relative abundances of specific bacteria.

HFD feedings has been associated with modification in the gut microbial profile. ${ }^{(33-35)}$ In this study, we observed a considerable alteration induced by HFD feeding, which was characterized by decreased alpha-diversity and decreased abundance of Phyla Deferribacteres and Proteobacteria (Fig. 3B-D and 4A). We further observed that FRB supplementation under the HFDfeeding condition modulated the gut microbiota in mice (Fig. 3D, 4, and 5). An extensive change in the ratio of Firmicutes/ Bacteroidetes may be associated with obesity and body weight loss upon dietary intervention. ${ }^{(21,36)}$ The dysbiosis in individuals with obesity is often characterized by a high Firmicutes/ Bacteroidetes ratio. ${ }^{(37)}$ In our results, the Firmicutes/Bacteroidetes ratio was significantly higher in the HFD group than in the HFD + FRB group at day 14 after the onset of the experiment. However, the values were similar in both groups at day 54 (Fig. 4F). Although whether the Firmicutes/Bacteroidetes ratio can be associated with determination of health status still remains controversial and, more specifically, is considered as a hallmark of obesity, ${ }^{(22)}$ it is notable that FRB supplementation induces such an alteration in the gut microbiota.

Studies have reported that $\mathrm{RB}$ can reduce body fat in obese rodents and humans. ${ }^{(38-42)} \gamma$-Oryzanol $(\mathrm{OZ})$ and the ferulic acid (FA) ester with phytosterols, primarily present in $\mathrm{RB}$, have been shown to improve markers of metabolic syndrome. ${ }^{(43)} \mathrm{FA}$ and $\mathrm{OZ}$ could be used as dietary supplements to alleviate the harmful effects of HFD. ${ }^{(43)}$ The finding that FRB administration did not significantly affect the appetite or behavioral activity of mice (Fig. 2C and 3), the mechanism of weight gain suppression by FRB, may be due to the host metabolic alteration induced by the biological function of FRB. Considering that the averaged consumption of FRB is limited in this experiment $(2.89 \times$ $10^{-3} \mathrm{~g} /$ day/mouse), the beneficial effect of the FRB should be dependent on its function as a multispecies probiotic. Our analyses demonstrated that Bacillus, Pediococcus, Staphylococcus, the unclassified order Streptophyta, Oryza, Enterococcus, and 21 other genera were detected in the final product of FRB (Table 2). We had anticipated that some of these bacteria should be detected in the feces of HFD + FRB mice. However, we detected only five genera (Enterococcus, Lactobacillus, Bacteroides, Prevotella, and the unclassified family Peptostreptococcaceae) among the bacteria contained in FRB in the feces of mice fed with HFD + FRB. In particular, the relative abundances of Lactobacillus and Prevotella appeared to be unaffected by FRB supplementation, and those of Enterococcus and the unclassified family Peptostreptococcaceae appeared to be even suppressed by FRB supplementation (Fig. 5). The results of a series of bacterial analyses, including PCoA, demonstrated that the FRB supplementation caused a considerable alteration in the gut microbiota in this experiment (Fig. 3-5). These data suggest that the intervention by the FRB in this experiment did not lead to the simple addition of the beneficial bacteria contained in FRB to the original host gut microbiota. It could also have induced host-microbiota interactions and bacterial interactions in the gut microbiota, resulting in a significant alteration in the state of the gut microbiota. Clostridium difficile is the pathogen responsible for a large population of diarrhea and colitis associated with antibiotic use. ${ }^{(44)}$ It has been reported that the probiotic strain Clostridium butyricum MIYAIRI 588 effectively prevents antibiotic-associated diarrhea in pediatric patients. ${ }^{(45)}$ C. butyricum MIYAIRI 588 significantly reduced the $C$. difficile toxin A detected in the feces of patients subject to Helicobacter pylori eradication with antibiotics. ${ }^{(46)}$ One of the mechanisms underlying the detoxification of $C$. difficile by C. butyricum MIYAIRI 588 is the inhibition of toxin-protein production based on the proximity or contact between the two species. ${ }^{(47)}$ Another example of the function of probiotics based on microbial interaction is that Saccharomyces boulardii protease inhibits the intestinal effects of $C$. difficile toxin A by proteolysis of the toxin and inhibition of toxin A binding to its receptor. ${ }^{(48)}$ Future studies must explore the type of interactions between microbes or between host and microbes underlying the function of FRB in the alteration of the gut microbiota.

Our analyses of OAs, including SCFAs, revealed that the fecal concentration of succinic acid was decreased in the HFD + FRB group compared with that in the HFD group (Fig. 6A). Because SCFAs and several OAs are the metabolites produced by the gut microbiota, the alteration in the gut microbiota is well reflected by the alteration in the fecal concentration of SCFAs and OAs relevant to the gut microbiome. ${ }^{(49,50)}$ Consistent with this relationship, our results also demonstrated that the fecal concentration of succinic acid correlated well with the relative abundances of Turicibacter, Enterococcus, and the unclassified family Peptostreptococcaceae (Fig. 6G and H). The relative abundances of these bacteria were induced by HFD feeding but suppressed by FRB supplementation (Fig. 5A, E, and 6H). A previous study showed that obesity was associated with elevated levels of circulating succinate. ${ }^{(51)}$ The authors of that study also found that this increase was associated with specific changes in the gut microbiota related to succinate metabolism, i.e., a higher relative abundance of succinate-producing Prevotellaceae and Veillonellaceae, and a lower relative abundance of succinate-consuming Odoribacteraceae and Clostridaceae in obese individuals. ${ }^{(51)}$ Circulating levels of succinic acid are elevated in several physiological conditions such as endurance exercise and a few metabolic- and inflammatory-related diseases, including ischemic heart disease, hypertension, type 2 diabetes, and obesity. ${ }^{(52)}$ Thus, succinate appears to be not merely a significant intermediate of the tricarboxylic acid cycle considered as a marker for pathophysiological conditions in the host but also a by-product of some bacteria and a primary cross-feeding metabolite between gut-resident microbes. ${ }^{(52)}$ These results indicate that the HFD-induced alteration in the gut microbiota was further modulated by FRB supplementation, which led to the suppressed level of succinic acid in feces.

We also detected the suppressed levels of glutamic acid, malic acid, sorbitol, uracil, and xylitol in the serum of mice fed with HFD + FRB compared with those of mice fed with HFD in the metabolome analyses (Fig. 7B and C). However, we detected an elevated level of fumaric acid in the serum of mice fed with HFD + FRB. Among these peripheral metabolites and fecal OAs, succinic acid, malic acid, fumaric acid, and glutamic acid are tricarboxylic acid cycle intermediates or relevant to the tricarboxylic acid cycle. Patel et al. ${ }^{(53)}$ have shown that the serum of HFD-fed mice displayed significantly reduced concentrations of glutamic acid, succinic acid, fumaric acid, and malic acid, suggesting that the anaplerosis for the tricarboxylic acid cycle was induced by HFD feeding. Park et al. ${ }^{(54)}$ have demonstrated that the concentrations of fumaric acid, sorbitol, and several sugars, including glucose, were significantly increased in the serum of the HFD group but decreased in that of the group administered with kimchi-derived bacteria, whereas serum malic acid concentration was significantly decreased in the HFD group but increased in the group intervened by kimchi-derived bacteria. Although our findings are partially consistent with these previous results, our findings suggest that HFD feeding induces metabolic impairment and enhanced blood sugar levels, which can be modified by FRB supplementation.

Several fermented foods such as kimchi and doenjang are known to have anti-obesity effects. ${ }^{(54-58)}$ The Korean fermented soybean paste "doenjang" could suppress body weight gain, 
reduce the amount of body fat, and improve the serum lipid profile in HFD-fed mice. ${ }^{(55)}$ It is generally accepted that the various microbial communities contained in these fermented materials function synergistically to exhibit efficacy against obesity. Kimchi is known to be abundant in probiotics such as Lactobacillus, Lactococcus, and Pediococcus that have antiobesity effects. ${ }^{(54)}$ Administration of the microbial community derived from kimchi has been found to decrease body weight, adipose tissue, and liver weight gains in HFD-fed mice. ${ }^{(54)}$ Considering the mechanisms underlying the benefits of these fermented foods, studies have often focused on specific types of beneficial bacteria such as Lactobacillus and Lactococcus. ${ }^{(2,59)}$ As described earlier, most bacteria contained in FRB were not detected in the feces of mice fed with HFD + FRB, and the relative abundances of some bacteria contained in FRB were even less in the gut microbiota of HFD + FRB mice than in the gut microbiota of HFD mice. These findings imply that our results have shed new insights on another aspect of the mechanisms of intervention of the gut microbiota by administering fermented products containing multispecies probiotics, in which hostmicrobiota interactions and bacterial interactions in the gut microbiota are triggered, rather than adding a few beneficial bacteria and the metabolic products of the beneficial bacteria to the host, resulting in the suppression of the bacteria with possible adverse effects on host health.

The primary BAs, including CA CDCA, UDCA, aMCA, and bMCA, in mice are synthesized from cholesterol in the liver. ${ }^{(32)}$ Before excretion into bile and further passage to the duodenum, BAs are almost exclusively conjugated with taurine or taurinederivatives, or, much less commonly, glycine in mice and rats. ${ }^{(32)}$ The gut microbiota modulates BA synthesis in the hepatocyte by regulating the expression of some of the enzymes involved in synthesizing BAs. ${ }^{(26,32)}$ After the secretion of conjugated BAs into the intestinal tract, they are modified by gut microbiota to produce secondary BAs, which lack taurine or glycine residues. ${ }^{(26,32)}$ Most BAs are actively reabsorbed by the ileum and enter the portal vein. ${ }^{(60)}$ The diversity of BAs in circulation is affected by their metabolism in the liver and intestinal bacteria. ${ }^{(60)}$ Therefore, the BAs synthesized by the host influence the intestinal bacterial composition, and the intestinal bacteria modify the circulating BA composition in the host. ${ }^{(26)}$ Although we did not observe apparent differences in the serum BA profiles between the HFD and HFD + RFB groups (Fig. 8), bacterial alterations are often reflected in the BA profiles. Golubeva et al. ${ }^{(61)}$ compared fecal and serum BA profiles in both wild-type and BTBR $\mathrm{T}^{+}$Itpr $3^{\mathrm{tf}} / \mathrm{J}$ mice, which are known to exhibit autism-like behavior with the gut microbiota being different from that in wild-type mice. Their data revealed considerable differences between fecal and serum BA profiles in each genotype. ${ }^{(61)}$ Selwyn et al. ${ }^{(60)}$ also reported that the BAs profiles in the serum, liver, bile, ileum, and feces differ between each other. Therefore, we must examine whether the BA profiles in fecal or other organ-derived samples reflect the alterations in the gut microbiota induced by FRB supplementation in our mouse model in future studies.

We acknowledge several limitations to our study. We did not use mice fed with a regular diet as a control. Mice at day 0 can be treated as such a regular diet-fed control, but we missed such a control in the metabolome and the BA analyses, which has limited the interpretation of our results in those analyses. The age-adjusted prevalence of severe obesity in adults was reported to be higher in women than in men in the United States. ${ }^{(62)}$ The causes of obesity are both biological and social and vary by sex or gender. ${ }^{(63)}$ It is necessary to assess whether the FRB has similar functions in male HFD-fed mice in future studies. We observed that the weight gain due to HFD feeding was suppressed by FRB supplementation, and the peripheral metabolomic profiles and fecal OA profiles were changed by FRB supplementation. However, we did not investigate whether such alterations in those metabolomic profiles and fecal OAs underlie the suppressed gain of body weight in the mice. Therefore, it is vital to explore the causal relationships between the peripheral metabolites, the fecal OAs, and the body weight gain in future studies.

\section{Author Contributions}

Conceived and designed the experiments: ST and HM. Performed the experiments: ST, YM, TK, TT, RS, TW, KM, and KA. Analyzed the data: ST. Wrote the paper: ST and HM. Supervised the study: ST, HM, and KM.

\section{Acknowledgments}

This work was supported by a grant for exploratory research from the Life Science Innovation Center at University of Fukui. In addition, we thank S. Kanae, M. Ishida, T. Nogi for technical assistance with the animal experiments.

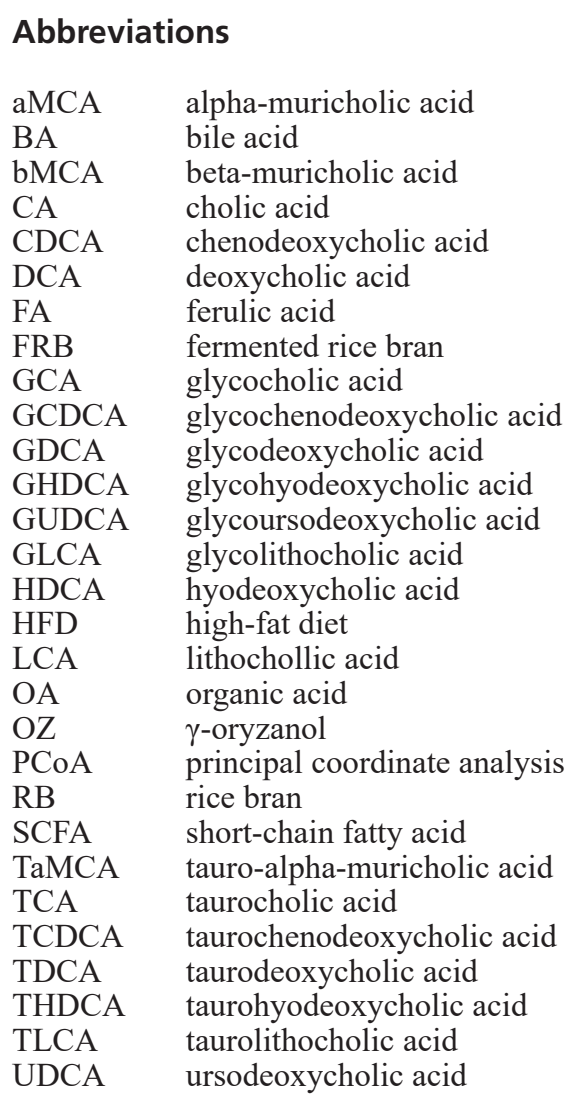

\section{Conflict of Interest}

No potential conflicts of interest were disclosed.

\section{References}

1 Minamiyama Y, Takemura S, Yoshikawa T, Okada S. Fermented grain products, production, properties and benefits to health. Pathophysiology
2003; 9: 221-227.

2 Shahbazi R, Sharifzad F, Bagheri R, Alsadi N, Yasavoli-Sharahi H, Matar C. 
Anti-inflammatory and immunomodulatory properties of fermented plant foods. Nutrients 2021; 13: 1516.

3 Wastyk HC, Fragiadakis GK, Perelman D, et al. Gut-microbiota-targeted diets modulate human immune status. Cell 2021; 184: 4137-4153.e14.

4 Choi JY, Paik DJ, Kwon DY, Park Y. Dietary supplementation with rice bran fermented with Lentinus edodes increases interferon- $\gamma$ activity without causing adverse effects: a randomized, double-blind, placebo-controlled, parallel-group study. Nutr J 2014; 13: 35.

5 Kumar A, Henderson A, Forster GM, et al. Dietary rice bran promotes resistance to Salmonella enterica serovar Typhimurium colonization in mice. BMC Microbiol 2012; 12: 71.

6 Alauddin M, Shirakawa H, Koseki T, et al. Fermented rice bran supplementation mitigates metabolic syndrome in stroke-prone spontaneously hypertensive rats. BMC Complement Altern Med 2016; 16: 442.

7 Islam J, Koseki T, Watanabe K, et al. Dietary supplementation of fermented rice bran effectively alleviates dextran sodium sulfate-induced colitis in mice. Nutrients 2017; 9: 747.

8 Pham T, Teoh KT, Savary BJ, Chen MH, McClung A, Lee SO. In vitro fermentation patterns of rice bran components by human gut microbiota. Nutrients 2017; 9: 1237.

9 Nealon NJ, Parker KD, Lahaie P, et al. Bifidobacterium longum-fermented rice bran and rice bran supplementation affects the gut microbiome and metabolome. Benef Microbes 2019; 10: 823-839.

10 Tochitani S, Ikeno T, Ito T, Sakurai A, Yamauchi T, Matsuzaki H. Administration of non-absorbable antibiotics to pregnant mice to perturb the maternal gut microbiota is associated with alterations in offspring behavior. PLoS One 2016; 11: e0138293.

11 Tochitani S, Furukawa T, Bando R, et al. GABAA receptors and maternally derived taurine regulate the temporal specification of progenitors of excitatory glutamatergic neurons in the mouse developing cortex. Cereb Cortex 2021; 31: 4554-4575.

12 Tsukahara $\mathrm{T}$, Inoue $\mathrm{R}$, Nakayama $\mathrm{K}$, Inatomi $\mathrm{T}$. Inclusion of Bacillus amyloliquefaciens strain TOA5001 in the diet of broilers suppresses the symptoms of coccidiosis by modulating intestinal microbiota. Anim Sci J 2018; 89: 679-687.

13 Inoue R, Sakaue Y, Sawai C, et al. A preliminary investigation on the relationship between gut microbiota and gene expressions in peripheral mononuclear cells of infants with autism spectrum disorders. Biosci Biotechnol Biochem 2016; 80: 2450-2458.

14 Inoue R, Sawai T, Sawai C, et al. A preliminary study of gut dysbiosis in children with food allergy. Biosci Biotechnol Biochem 2017; 81: 2396-2399.

15 Lee DH, Zo YG, Kim SJ. Nonradioactive method to study genetic profiles of natural bacterial communities by PCR-single-strand-conformation polymorphism. Appl Environ Microbiol 1996; 62: 3112-3120.

16 Tsukahara $\mathrm{T}$, Matsukawa $\mathrm{N}$, Tomonaga $\mathrm{S}$, Inoue $\mathrm{R}$, Ushida $\mathrm{K}$, Ochiai $\mathrm{K}$. High-sensitivity detection of short-chain fatty acids in porcine ileal, cecal, portal and abdominal blood by gas chromatography-mass spectrometry. Anim Sci J 2014; 85: 494-498.

17 Tomonaga S, Okuyama H, Tachibana T, Makino R. Effects of high ambient temperature on plasma metabolomic profiles in chicks. Anim Sci J 2018; 89: 448-455.

18 Hagio M, Matsumoto M, Fukushima M, Hara H, Ishizuka S. Improved analysis of bile acids in tissues and intestinal contents of rats using LC/ESI-MS. $J$ Lipid Res 2009; 50: 173-180.

19 Lovejoy JC, Sainsbury A; Stock Conference Working Group. Sex differences in obesity and the regulation of energy homeostasis. Obes Rev 2009; 10: 154 167.

20 Ley RE, Turnbaugh PJ, Klein S, Gordon JI. Microbial ecology: human gut microbes associated with obesity. Nature 2006; 444: 1022-1023.

21 Turnbaugh PJ, Ley RE, Mahowald MA, Magrini V, Mardis ER, Gordon JI. An obesity-associated gut microbiome with increased capacity for energy harvest. Nature 2006; 444: 1027-1031.

22 Magne F, Gotteland M, Gauthier L, et al. The Firmicutes/Bacteroidetes ratio: a relevant marker of gut dysbiosis in obese patients? Nutrients 2020; 12: 1474.

23 Clemente JC, Ursell LK, Parfrey LW, Knight R. The impact of the gut microbiota on human health: an integrative view. Cell 2012; 148: 1258-1270.

24 Kimura I, Inoue D, Maeda $\mathrm{T}$, et al. Short-chain fatty acids and ketones directly regulate sympathetic nervous system via $\mathrm{G}$ protein-coupled receptor 41 (GPR41). Proc Natl Acad Sci U S A 2011; 108: 8030-8035.
25 Kimura I, Ichimura A, Ohue-Kitano R, Igarashi M. Free fatty acid receptors in health and disease. Physiol Rev 2020; 100: 171-210.

26 Tochitani S. Vertical transmission of gut microbiota: points of action of environmental factors influencing brain development. Neurosci Res 2021; 168: 83-94.

27 Brown AJ, Goldsworthy SM, Barnes AA, et al. The Orphan G proteincoupled receptors GPR41 and GPR43 are activated by propionate and other short chain carboxylic acids. J Biol Chem 2003; 278: 11312-11319.

28 Kimura I, Ozawa K, Inoue D, et al. The gut microbiota suppresses insulinmediated fat accumulation via the short-chain fatty acid receptor GPR43. Nat Commun 2013; 4: 1829 .

29 Lin HV, Frassetto A, Kowalik EJ Jr, et al. Butyrate and propionate protect against diet-induced obesity and regulate gut hormones via free fatty acid receptor 3-independent mechanisms. PLoS One 2012; 7: e35240.

30 den Besten G, Bleeker A, Gerding A, et al. Short-chain fatty acids protect against high-fat diet-induced obesity via a PPAR $\gamma$-dependent switch from lipogenesis to fat oxidation. Diabetes 2015; 64: 2398-2408.

31 Lu Y, Fan C, Li P, Lu Y, Chang X, Qi K. Short chain fatty acids prevent high-fat-diet-induced obesity in mice by regulating $\mathrm{G}$ protein-coupled receptors and gut microbiota. Sci Rep 2016; 6: 37589.

32 Wahlström A, Sayin SI, Marschall HU, Bäckhed F. Intestinal crosstalk between bile acids and microbiota and its impact on host metabolism. Cell Metab 2016; 24: 41-50.

33 Zhang C, Zhang M, Pang X, Zhao Y, Wang L, Zhao L. Structural resilience of the gut microbiota in adult mice under high-fat dietary perturbations. ISME $J$ 2012; 6: 1848-1857.

34 Daniel H, Gholami AM, Berry D, et al. High-fat diet alters gut microbiota physiology in mice. ISME J 2014; 8: 295-308.

35 Murphy EA, Velazquez KT, Herbert KM. Influence of high-fat diet on gut microbiota: a driving force for chronic disease risk. Curr Opin Clin Nutr Metab Care 2015; 18: 515-520.

36 Ley RE, Peterson DA, Gordon JI. Ecological and evolutionary forces shaping microbial diversity in the human intestine. Cell 2006; 124: 837-848.

37 Castaner O, Goday A, Park YM, et al. The gut microbiome profile in obesity: a systematic review. Int J Endocrinol 2018; 2018: 4095789.

38 Hongu N, Kitts DD, Zawistowski J, et al. Pigmented rice bran and plant sterol combination reduces serum lipids in overweight and obese adults. $J$ Am Coll Nutr 2014; 33: 231-238.

39 Boonloh K, Kukongviriyapan V, Kongyingyoes B, Kukongviriyapan U, Thawornchinsombut S, Pannangpetch P. Rice bran protein hydrolysates improve insulin resistance and decrease pro-inflammatory cytokine gene expression in rats fed a high carbohydrate-high fat diet. Nutrients 2015; 7: 6313-6329.

40 Justo ML, Claro C, Zeyda M, Stulnig TM, Herrera MD, Rodríguez-Rodríguez R. Rice bran prevents high-fat diet-induced inflammation and macrophage content in adipose tissue. Eur J Nutr 2016; 55: 2011-2019.

41 Yang H, Yoon M, Um MY, et al. Sleep-promoting effects and possible mechanisms of action associated with a standardized rice bran supplement. Nutrients 2017; 9: 512.

42 Zou Y, Ju X, Chen W, et al. Rice bran attenuated obesity via alleviating dyslipidemia, browning of white adipocytes and modulating gut microbiota in high-fat diet-induced obese mice. Food Funct 2020; 11: 2406-2417.

43 Wang O, Liu J, Cheng Q, et al. Effects of ferulic acid and $\gamma$-oryzanol on high-fat and high-fructose diet-induced metabolic syndrome in rats. PLoS One 2015; 10: e0118135.

44 Leffler DA, Lamont JT. Clostridium difficile infection. N Engl J Med 2015; 373: $287-288$

45 Seki H, Shiohara M, Matsumura T, et al. Prevention of antibiotic-associated diarrhea in children by Clostridium butyricum MIYAIRI. Pediatr Int 2003; 45: 86-90.

46 Imase K, Takahashi M, Tanaka A, et al. Efficacy of Clostridium butyricum preparation concomitantly with Helicobacter pylori eradication therapy in relation to changes in the intestinal microbiota. Microbiol Immunol 2008; 52: 156-161.

47 Woo TDH, Oka K, Takahashi M, et al. Inhibition of the cytotoxic effect of Clostridium difficile in vitro by Clostridium butyricum MIYAIRI 588 strain. $J$ Med Microbiol 2011; 60 (Pt 11): 1617-1625.

48 Castagliuolo I, LaMont JT, Nikulasson ST, Pothoulakis C. Saccharomyces boulardii protease inhibits Clostridium difficile toxin A effects in the rat ileum. Infect Immun 1996; 64: 5225-5232. 
49 Dalile B, Van Oudenhove L, Vervliet B, Verbeke K. The role of short-chain fatty acids in microbiota-gut-brain communication. Nat Rev Gastroenterol Hepatol 2019; 16: 461-478.

50 Silva YP, Bernardi A, Frozza RL. The role of short-chain fatty acids from gut microbiota in gut-brain communication. Front Endocrinol (Lausanne) 2020; 11: 25 .

51 Serena C, Ceperuelo-Mallafré V, Keiran N, et al. Elevated circulating levels of succinate in human obesity are linked to specific gut microbiota. ISME J 2018; 12: 1642-1657.

52 Fernández-Veledo S, Vendrell J. Gut microbiota-derived succinate: friend or foe in human metabolic diseases? Rev Endocr Metab Disord 2019; 20: 439447.

53 Patel DP, Krausz KW, Xie C, Beyoğlu D, Gonzalez FJ, Idle JR. Metabolic profiling by gas chromatography-mass spectrometry of energy metabolism in high-fat diet-fed obese mice. PLoS One 2017; 12: e0177953.

54 Park SE, Kwon SJ, Cho KM, et al. Intervention with kimchi microbial community ameliorates obesity by regulating gut microbiota. $J$ Microbiol 2020; 58: 859-867.

55 Park NY, Rico CW, Lee SC, Kang MY. Comparative effects of doenjang prepared from soybean and brown rice on the body weight and lipid metabolism in high fat-fed mice. J Clin Biochem Nutr 2012; 51: 235-240.

56 Kim EK, An SY, Lee MS, et al. Fermented kimchi reduces body weight and improves metabolic parameters in overweight and obese patients. Nutr Res 2011; 31: 436-443.

57 Park JA, Tirupathi Pichiah PB, Yu JJ, Oh SH, Daily JW 3rd, Cha YS. Anti- obesity effect of kimchi fermented with Weissella koreensis OK1-6 as starter in high-fat diet-induced obese C57BL/6J mice. J Appl Microbiol 2012; 113: $1507-1516$.

58 An SY, Lee MS, Jeon JY, et al. Beneficial effects of fresh and fermented kimchi in prediabetic individuals. Ann Nutr Metab 2013; 63: 111-119.

59 Kadooka Y, Sato M, Imaizumi K, et al. Regulation of abdominal adiposity by probiotics (Lactobacillus gasseri SBT2055) in adults with obese tendencies in a randomized controlled trial. Eur J Clin Nutr 2010; 64: 636-643.

60 Selwyn FP, Csanaky IL, Zhang Y, Klaassen CD. Importance of large intestine in regulating bile acids and glucagon-like peptide-1 in germ-free mice. Drug Metab Dispos 2015; 43: 1544-1556.

61 Golubeva AV, Joyce SA, Moloney G, et al. Microbiota-related changes in bile acid \& tryptophan metabolism are associated with gastrointestinal dysfunction in a mouse model of autism. EBioMedicine 2017; 24: 166-178.

62 Hales CM, Carroll MD, Fryar CD, Ogden CL. Prevalence of obesity and severe obesity among adults: United States, 2017-2018. NCHS Data Brief 2020; (360): 1-8.

63 Kanter R, Caballero B. Global gender disparities in obesity: a review. $A d v$ Nutr 2012; 3: 491-498.

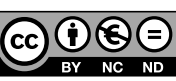

This is an open access article distributed under the terms of the Creative Commons Attribution-NonCommercial-NoDerivatives License (http://creativecommons.org/licenses/by-nc-nd/4.0/). 